\title{
A Nonlinear Vibration Isolator Achieving High-Static-Low-Dynamic Stiffness and Tunable Anti-resonance Frequency Band
}

\author{
Xiuting Sun ${ }^{1,2}$, Xingjian Jing ${ }^{2 *}$ \\ 1 School of Mechanical Engineering, University of Shanghai for Science and Technology, Shanghai, PRC \\ 2 Department of Mechanical Engineering, The Hong Kong Polytechnic University, Hong Kong, PRC \\ * The corresponding author, Email: xingjian.jing@ polyu.edu.hk
}

\begin{abstract}
This study investigates theoretically and experimentally a vibration isolator constructed by an n-layer Scissor-Like Structure (SLS), focusing on the analysis and design of nonlinear stiffness and damping characteristics for advantageous isolation performance in both orthogonal directions. With the mathematical modeling, the influence incurred by different structural parameters on system isolation performance is studied. It is shown that, (a) nonlinear high-static-low-dynamic stiffness and damping characteristics can be seen such that the system can achieve good isolation performance in both directions, (b) an anti-resonance frequency band exists due to the coupling effect between the linear and nonlinear stiffness in the two orthogonal directions within the structure, and (c) all these performances are designable with several structural parameters. The advantages of the proposed system are shown through comparisons with an existing quasi-zero-stiffness vibration isolator (QZS-VI) and a traditional mass-spring-damper vibration isolator (MSD-VI), and further validated by experimental results.
\end{abstract}

Keywords: Vibration isolation; Nonlinear stiffness and damping; Structural nonlinearity; Anti-resonance 


\section{Introduction}

The vibration isolation at low frequency is a key issue in various engineering practice, including protection of precision instruments [1], vibration isolation for multi-layer building [2], vibration isolation in space launch and/or on-orbit operations [3-4], and vibration control in vehicle suspension systems [5] etc. The structures consisting of springs and dampers are often used for vibration isolation of frequencies $\sqrt{2}$ times larger than the natural frequency of the system.

To suppress low frequency vibration, a Quasi-Zero-Stiffness Vibration Isolator (QZS-VI) is studied in recent years [6-15], which can achieve ultra-low dynamic stiffness. However, to guarantee loading capacity but keeping the QZS property simultaneously (i.e., a high-static-low-frequency stiffness), the nonlinear stiffness of the system should be increased and structural parameters should also be chosen properly [12-13], [15]. Therefore, parametric excitation and active control are studied to improve the performance of QZS systems [16-18]. Because the equivalent stiffness of the QZS-VI mentioned above is like a Duffing oscillator having strong nonlinearity, a multi-steady-state frequency region is often inevitable, which induces stability problem around the equilibrium [19-21]. Thus, it is difficult to minimize the magnitude at multi-steady-state frequency band and reduce the natural frequency while keeping the loading capacity at the same time in the QZS-VIs.

Some other typical passive vibration isolation methods are to utilize an additional system as vibration absorbers to transmit vibration energy to the additional system by adjusting its natural frequency [22-28]. The vibration absorber can be designed to change the resonant peak to an anti-resonance region since the additional system is coupled with the master structure. The larger is the difference between the natural frequencies of the master and absorber systems, the wider the 
anti-resonance frequency band is. However, a vibration absorber can only work when its natural frequency is close to a desired ratio of the natural frequency of the master structure [22-26]. This means that vibration absorbers will lose effectiveness in vibration suppression when the characteristics of master structures change.

To obtain better isolation and stability effects, scissor-jack structures, consisting of rods and joints, are proposed and studied in buildings [30-32], vehicle seats [33-34] and isolation platforms [35-36]. A scissor structure is used as a foldable variable stiffness spring (FVSS) with viscous dampers to increases the damping effect in 1D vibration control [30-32]. In Ref [32], it is shown that the system damping property can be optimized and improved with only using linear viscous dampers within the scissor structure. In Refs [33-34], air springs are applied in a one-layer scissor structure as a seat suspension system to supply active control force that is needed for vibration isolation. All these results mentioned above actually investigate only the damping design with different scissor-like supporting structures. Utilizing the scissor-like element (SLE) as a vibration isolation component is proposed and analyzed in [35] but with only simulation results for harmonic base excitations. A general n-layer scissor-like structured (SLS) platform for 1-direction (1-D) vibration isolation is systematically studied in [36] for exploring the design of nonlinear high-static-low-dynamic stiffness. In [36-41], mathematical modeling, frequency domain analysis and design of structural or characteristic parameters are studied and discussed for exploring beneficial nonlinear stiffness and damping properties in vibration control.

In this study, the n-layer SLS platform is employed for constructing a 2-direction (2-D) vibration isolation system by relaxing the horizontal motion, and thus to achieve vibration isolation in two orthogonal directions simultaneously. A similar way can be used to construct a 3D or above 
vibration isolation platform. It is shown that there is an anti-resonance frequency band due to the coupling effect between the vertical and horizontal stiffness, which demonstrates much better isolation effect than traditional mass-spring-damper vibration isolation (MSD-VI) systems and the QZS-VIs mentioned above. By designing structural parameters, good quasi-zero stiffness property can be achieved without the disadvantages of QZS-VI systems. Experimental results are given to vindicate the advantageous high-static-low-dynamic stiffness and beneficial nonlinear damping property of the proposed system.

The contributions of this study lies in the following points. (a) A passive 2-D vibration isolator is proposed, which is shown to have advantageous vibration isolation performance compared with some existing systems; (b) The 2-D SLS-based platform is demonstrated to be a new and effective method for achieving quasi-zero stiffness and anti-resonance property simultaneously; (c) Experimental studies are conducted which demonstrate the advantages of the proposed isolation system as a versatile platform for realization of beneficial nonlinear stiffness and damping in practice.

The paper is organized as follows. The schematic structure of an n-layer SLS platform is introduced in Section 2 and a mathematical modeling is conducted here. Then the nonlinear stiffness and damping properties under different structural parameters are studied in Section 3. Comparisons of vibration isolation performance with traditional MSD-VI, and existing QZS-VI systems are discussed in Section 4 and Section 5, respectively. Then an experimental prototype is conducted to validate the isolation effect in Section 6. A conclusion is drawn thereafter.

\section{The 2-D n-layer SLS-based platform and its modeling}




\subsection{The 2-D platform}

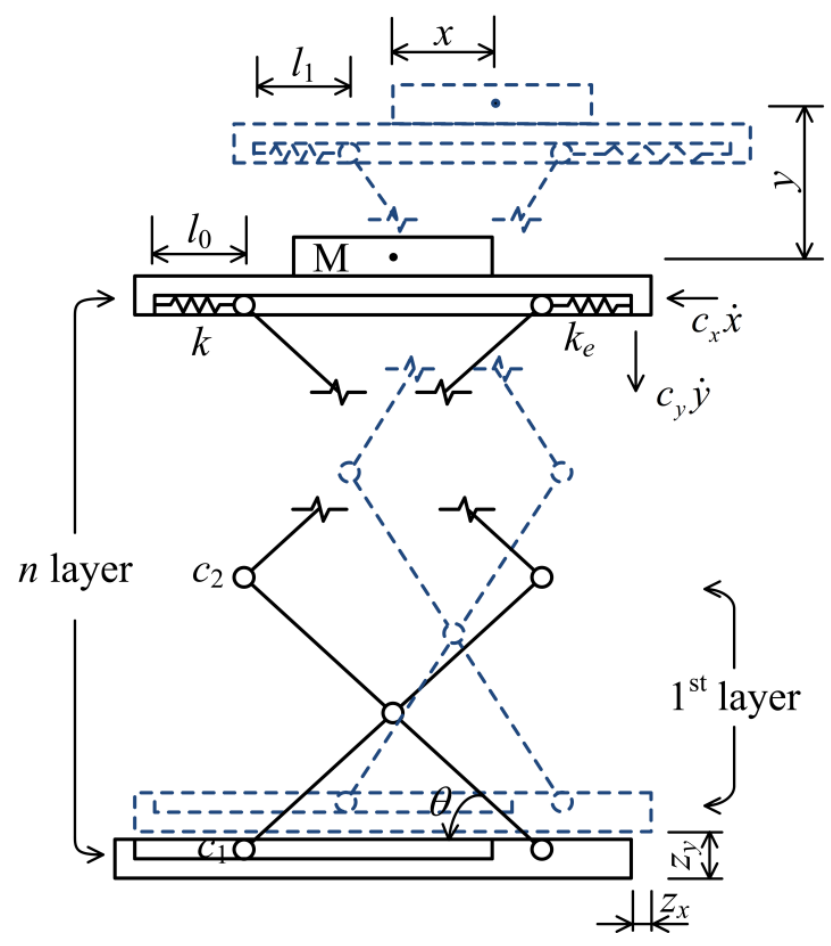

Fig. 1 The n-layer SLS-based platform for 2-D base excitations. Solid lines show the original state and dashed lines show the vibration state.

Fig. 1 is the front view diagram of the 2-D SLS-based platform. The bottom layer is defined as the first SLS layer as shown in Fig. 1 and the layer number of the SLS is counted from bottom to top. For two-orthogonal-direction isolation (in horizontal and vertical), the left and right joints at the top layer and the left joint at the bottom layer can freely slide along pre-designed horizontal tracks. The mass of the isolation object is denoted by $M$. Two linear springs whose stiffness is $k$ and $k_{e}$ are assembled in the left and right tracks at the top layer of the platform. The contacting surface on these sliding tracks and in rotating joints can be designed for different friction properties. In this paper, the viscous friction is adopted with friction coefficient $c_{1}$ for the horizontal motion and $c_{2}$ for the rotational motion. The connecting rods have the same length i.e., $2 l$ and the assembly angle with respect to the horizontal line is given by $\theta$ (corresponding to the springs at the free state). The air 
damping coefficients in horizontal and vertical are $c_{x}$ and $c_{y}$, respectively. All the structural parameters are listed in Table 1 in Appendix.

The 2-D base excitations are denoted by $z_{x}$ and $z_{y}$, respectively. The absolute motion in the horizontal direction is $x$; in the vertical is $y$ and the rotation motion of the connecting rods is $\varphi$. The material used in the isolation system is uniform and the horizontal and vertical motions of arbitrary points on the platform are the same denoted by $x$ and $y$. The relative motions are defined as $\hat{x}$ $=x-z_{x}$ and $\hat{y}=y-z_{y}$. All the variables are listed in Table 2 in Appendix. The geometrical relation of connecting rods and relative motions are shown in Fig. 2.

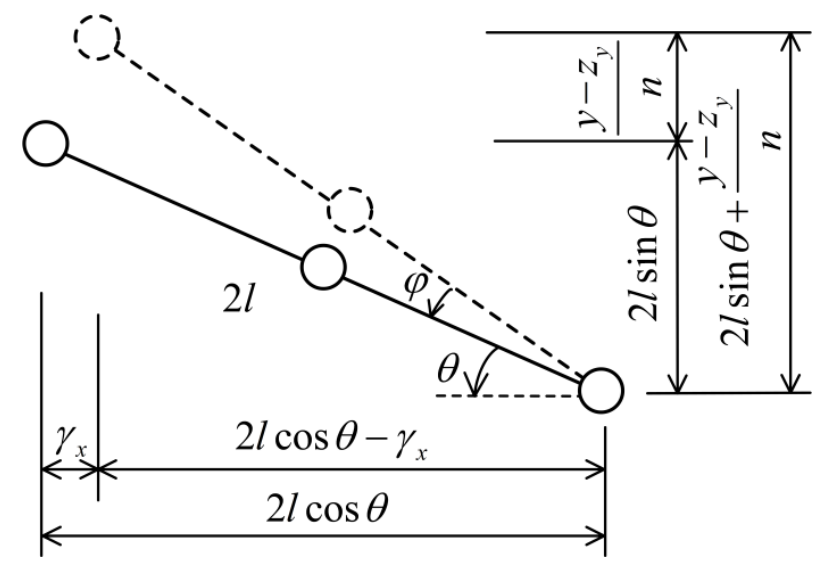

Fig. 2 The motion of a connecting rod in the first layer and geometrical relations. Solid lines for original state and dashed lines for the vibration state.

In Fig. 2, the horizontal motion of joints at the top and bottom layers denoted by $\gamma_{x}$ and the rotation motion of each rod denoted by $\varphi$ are given by

$$
\begin{aligned}
& \gamma_{x}=2 l \cos \left(\theta+\varphi_{0}\right)-\sqrt{(2 l)^{2}-\left(2 l \sin \left(\theta+\varphi_{0}\right)+y / n\right)^{2}} \\
& \varphi=\arctan \left(\frac{2 l \sin \left(\theta+\varphi_{0}\right)+y / n}{2 l \cos \left(\theta+\varphi_{0}\right)-\gamma_{x}}\right)-\theta-\varphi_{0}
\end{aligned}
$$

where $\gamma_{0}$ and $\varphi_{0}$ reflect the original static deformation due to the loading mass as 


$$
\begin{aligned}
& \gamma_{0}=2 l \cos \theta-\sqrt{(2 l)^{2}-\left(2 l \sin \theta+y_{0} / n\right)^{2}} \\
& \varphi_{0}=\arctan \left(\frac{\left.2 l \sin \theta+\frac{y_{0} / n}{2 l \cos \theta-\gamma_{0}}\right)-\theta}{2}\right.
\end{aligned}
$$

where $y_{0}$ is the static vertical deformation induced by loading $M g$. The equations above indicate that the motion through the n-layer SLS is only 1-DOF since the horizontal motion $\gamma_{x}$ and rotational motion $\varphi$ can both be expressed by the relative motion $\hat{y}$ and vertical direction distance induced by loading mass. The absolute motions of the isolation object $x$ and $y$ are chosen as generalized coordinate.

Although the modeling of the SLS platform has been studied in [36], the modeling of the 2-D SLS based platform here is not a trivial extension from the previous one due to the coupling effects between the vertical and horizontal stiffness of the system. Also, because of this coupling effect, the influence on isolation performance of each structural parameter would also be very different.

\subsection{Modeling}

The kinetic energy $T$ of the system is given by

$$
T=\frac{1}{2} M \dot{x}^{2}+\frac{1}{2} M \dot{y}^{2}
$$

The potential energy $V$ is the elastic energy of the two springs at the top layer in the horizontal direction, and the potential energy due to the gravity force, which can be expressed as

$$
\begin{aligned}
V & =\frac{1}{2} k\left(l_{1}-l_{0}\right)^{2}+\frac{1}{2} k_{e}\left(x-z_{x}+x_{0}\right)^{2}+M g\left(y_{0}+y\right) \\
& =\frac{1}{2} k\left[\gamma_{x}-\left(x-z_{x}\right)+\gamma_{0}-x_{0}\right]^{2}+\frac{1}{2} k_{e}\left(x-z_{x}+x_{0}\right)^{2}+M g\left(y_{0}+y\right)
\end{aligned}
$$

where $y_{0}$ is the vertical direction offset at zero equilibrium induced by static loading of $M g$. When the platform is in the static state, the gravitational energy is $V_{g}=M g y_{0}$ and the elastic potential energy of the spring deformation is $V_{e}=\frac{1}{2} k\left(\gamma_{0}-x_{0}\right)^{2}+\frac{1}{2} k_{e} x_{0}^{2}$ where $x_{0}$ is the horizontal static 
offset in the top spring $k_{\mathrm{e}}$ of the platform for static loading $M g$. Therefore, based on the minimum potential energy principle, it can be obtained

$$
\left\{\begin{array}{l}
\frac{\partial V_{e}}{\partial x_{0}}=\left(k+k_{e}\right) x_{0}-k\left[2 l \cos \theta-\sqrt{4 l^{2}-\left(y_{0} / n+2 l \sin \theta\right)^{2}}\right]=0 \\
\frac{\partial V_{e}}{\partial y_{0}}+\frac{\partial V_{g}}{\partial y_{0}}=k\left(\gamma_{0}-x_{0}\right) \frac{\partial \gamma_{0}}{\partial y_{0}}+M g=\frac{k\left(\gamma_{0}-x_{0}\right)\left(y_{0}+2 n l \sin \theta\right)}{n \sqrt{4 n^{2} l^{2}-\left(y_{0}+2 n l \sin \theta\right)^{2}}}+M g=0
\end{array}\right.
$$

From (4b), it can be obtained that

$$
\left\{\begin{array}{l}
k_{e} x_{0}+k\left(x_{0}-\gamma_{0}\right)=0 \\
k\left(\gamma_{0}-x_{0}\right) \frac{\tan \left(\theta+\varphi_{0}\right)}{n}+M g=0
\end{array}\right.
$$

The friction forces in the coulomb form can be minimized via surface-smoothing or material selection etc. This study considers only viscous friction for convenience in discussion. There are three horizontal tracks and $6 n$ joints in the SLS platform. The friction force of the horizontal track at the bottom layer is denoted by $F_{f x 1}$; the friction forces at the left and right sides of the top layer by $F_{f x 2}$ and $F_{f x 3}$ respectively; the rotational motion $\varphi$ of each connecting joint is denoted by $F_{f \varphi}$. The friction forces $F_{f x 1}, F_{f x 2}, F_{f x 3}$ and $F_{f \varphi}$ are given by

$$
\begin{gathered}
F_{f x 1}=-c_{1} \dot{\gamma}_{x} \\
F_{f x 2}=-c_{1}\left(\dot{\hat{x}}-\dot{\gamma}_{x}\right) \\
F_{f x 3}=-c_{1} \dot{\hat{x}} \\
F_{f \varphi}=-c_{2} \dot{\varphi}
\end{gathered}
$$

where $c_{1}$ and $c_{2}$ are the corresponding friction coefficients. With the generalized coordinates chosen as $(x, y)$, the generalized forces $Q x$ and $Q_{y}$ are given by

$$
Q_{x}=\sum \vec{F}_{i} \cdot \frac{\partial \vec{r}_{i}}{\partial x}=-c_{x} \dot{\hat{x}}-F_{f x 1} \cdot \frac{\partial \gamma_{x}}{\partial x}-F_{f x 2} \cdot \frac{\partial\left(\hat{x}-\gamma_{x}\right)}{\partial x}-F_{f x 3} \cdot \frac{\partial \hat{x}}{\partial x}-6 n F_{f \varphi} \cdot \frac{\partial \varphi}{\partial x}
$$

and 


$$
Q_{y}=\sum \vec{F}_{i} \cdot \frac{\partial \vec{r}_{i}}{\partial y}=-c_{y} \dot{\hat{y}}-F_{f x 1} \cdot \frac{\partial \gamma_{x}}{\partial y}-F_{f x 2} \cdot \frac{\partial\left(\hat{x}-\gamma_{x}\right)}{\partial y}-F_{f x 3} \cdot \frac{\partial \hat{x}}{\partial y}-6 n F_{f \varphi} \cdot \frac{\partial \varphi}{\partial y}
$$

where $\vec{F}_{i}$ denotes each force considered and $\vec{r}_{i}$ the direction of each force $F_{i}$. Substituting friction forces (5)-(8) into generalized forces (9)-(10), and applying the Lagrange principle yield

$$
\left\{\begin{array}{l}
\frac{\mathrm{d}}{\mathrm{d} t}\left(\frac{\partial L}{\partial \dot{x}}\right)-\frac{\partial L}{\partial x}=-c_{x} \dot{\hat{x}}-c_{1} \dot{\gamma}_{x} \cdot \frac{\partial \gamma_{x}}{\partial x}-c_{1}\left(\dot{\hat{x}}-\dot{\gamma}_{x}\right) \cdot \frac{\partial\left(\hat{x}-\gamma_{x}\right)}{\partial x}-c_{1} \dot{\hat{x}} \cdot \frac{\partial \hat{x}}{\partial x}-6 n c_{2} \dot{\varphi} \cdot \frac{\partial \varphi}{\partial x} \\
\frac{\mathrm{d}}{\mathrm{d} t}\left(\frac{\partial L}{\partial \dot{y}}\right)-\frac{\partial L}{\partial y}=-c_{y} \dot{\hat{y}}-c_{1} \dot{\gamma}_{x} \cdot \frac{\partial \gamma_{x}}{\partial y}-c_{1}\left(\dot{\hat{x}}-\dot{\gamma}_{x}\right) \cdot \frac{\partial\left(\hat{x}-\gamma_{x}\right)}{\partial y}-c_{1} \dot{\hat{x}} \cdot \frac{\partial \hat{x}}{\partial y}-6 n c_{2} \dot{\varphi} \cdot \frac{\partial \varphi}{\partial y}-M g
\end{array}\right.
$$

where $\dot{\varphi}=(\mathrm{d} \varphi / \mathrm{d} \hat{y}) \cdot \dot{\hat{y}}, \quad \dot{\gamma}_{x}=\left(\mathrm{d} \gamma_{x} / \mathrm{d} \hat{y}\right) \cdot \dot{\hat{y}}$. Substituting the kinetic energy (3), potential energy (4), and transport motion (1)-(2) into (11a), the dynamic equation of the 2-D n-layer SLS-based platform can be obtained as

$$
\left\{\begin{array}{l}
M \ddot{x}+\left[k\left(\hat{x}-\gamma_{x}-\gamma_{0}+x_{0}\right)+k_{e}\left(\hat{x}+x_{0}\right)\right]=-c_{x} \dot{\hat{x}}-c_{1}\left(\dot{\hat{x}}-\frac{\tan \left(\theta+\varphi_{0}+\varphi\right)}{n} \dot{\hat{y}}\right)-c_{1} \dot{\hat{x}} \\
M \ddot{y}+k\left(\hat{x}-\gamma_{x}-\gamma_{0}+x_{0}\right) \frac{\tan \left(\theta+\varphi_{0}+\varphi\right)}{n}=-c_{y} \dot{\hat{y}}-2 c_{1}\left(\frac{\tan \left(\theta+\varphi_{0}+\varphi\right)}{n}\right)^{2} \dot{\hat{y}} \\
\quad+c_{1} \frac{\tan \left(\theta+\varphi_{0}+\varphi\right)}{n} \dot{\hat{x}}-6 n c_{2}\left(\frac{\partial \varphi}{\partial y}\right)^{2} \dot{\hat{y}}-M g
\end{array}\right.
$$

Substituting $\gamma_{x}, \varphi$ into Eq. (11b), it has 


$$
\begin{aligned}
M \ddot{x}+ & {\left[k\left(\hat{x}-2 l \cos \left(\theta+\varphi_{0}\right)+\sqrt{(2 l)^{2}-\left(2 l \sin \left(\theta+\varphi_{0}\right)+y / n\right)^{2}}\right)+k\left(x_{0}-\gamma_{0}\right)+k_{e} \hat{x}+k_{e} x_{0}\right] } \\
& =-c_{x} \dot{\hat{x}}-c_{1}\left(\dot{\hat{x}}-\frac{2 l \sin \left(\theta+\varphi_{0}\right)+y / n}{n\left(2 l \cos \left(\theta+\varphi_{0}\right)-\gamma_{x}\right)} \dot{\hat{y}}\right)-c_{1} \dot{\hat{x}} \\
M \ddot{y}+ & k \frac{\left(y / n+2 l \sin \left(\theta+\varphi_{0}\right)\right)\left(-\hat{x}+\gamma_{0}-x_{0}+2 l \cos \left(\theta+\varphi_{0}\right)-\sqrt{(2 l)^{2}-\left(2 l \sin \left(\theta+\varphi_{0}\right)+y / n\right)^{2}}\right)}{n \sqrt{(2 l)^{2}-\left(2 l \sin \left(\theta+\varphi_{0}\right)+y / n\right)^{2}}} \\
& =-c_{y} \dot{\hat{y}}-2 c_{1}\left(\frac{2 l \sin \left(\theta+\varphi_{0}\right)+y / n}{n\left(2 l \cos \left(\theta+\varphi_{0}\right)-\gamma_{x}\right)}\right)^{2} \dot{\hat{y}}+c_{1} \frac{2 l \sin \left(\theta+\varphi_{0}\right)+y / n}{n\left(2 l \cos \left(\theta+\varphi_{0}\right)-\gamma_{x}\right)} \dot{\hat{x}} \\
& -6 n c_{2}\left(\frac{1}{\left.n \sqrt{(2 l)^{2}-\left(2 l \sin \left(\theta+\varphi_{0}\right)+y / n\right.}\right)^{2}}\right)^{2}
\end{aligned}
$$

It can be verified that around the equilibrium of this system, the system has weak nonlinearity both in stiffness and damping. As shown in Refs. [14] and [36], the nonlinearities incurred by the SLS can be accurately described by a third-order Taylor series for vibration in an appropriate range such as $[-0.1,0.1](\mathrm{m})$. The nonlinear terms obtained by the differential calculation of each term in Eq. (11c) can thus be expanded by Taylor series around the equilibrium. Then the terms representing the resilience are defined as the equivalent stiffness property and the ones representing the friction and damping are defined as the equivalent damping property [8]-[16], [27], [36], [40]. Therefore, expanding the stiffness and damping with Taylor series, the dynamic equations of the 2-D SLS-based platform in the two directions are 


$$
\left\{\begin{array}{l}
M \ddot{\hat{x}}+\left(k+k_{e}\right) x-k\left[\frac{\tan \left(\theta+\varphi_{0}\right)}{n} \hat{y}+\frac{\sec ^{3}\left(\theta+\varphi_{0}\right)}{4 n^{2}} \hat{y}^{2}+\frac{\sec ^{4}\left(\theta+\varphi_{0}\right) \tan \left(\theta+\varphi_{0}\right)}{8 l^{2} n^{3}} \hat{y}^{3}\right] \\
+\left(c_{x}+2 c_{1}\right) \dot{\hat{x}}-c_{1}\left[\frac{\tan \left(\theta+\varphi_{0}\right)}{n}+\frac{\sec ^{3}\left(\theta+\varphi_{0}\right)}{2 l n^{2}} \hat{y}+\frac{3 \sec ^{4}\left(\theta+\varphi_{0}\right) \tan \left(\theta+\varphi_{0}\right)}{8 l^{2} n^{3}} \hat{y}^{2}\right] \dot{\hat{y}}=-M \ddot{z}_{x}, \\
M \ddot{\hat{y}}+k\left[\frac{\tan ^{2}\left(\theta+\varphi_{0}\right)}{n^{2}} \hat{y}+\frac{3 \sec ^{3}\left(\theta+\varphi_{0}\right) \tan \left(\theta+\varphi_{0}\right)}{4 \ln ^{3}} \hat{y}^{2}+\frac{\left(3-2 \cos 2\left(\theta+\varphi_{0}\right)\right) \sec ^{6} \theta}{8 l^{2} n^{4}} \hat{y}^{3}\right]+c_{y} \dot{\hat{y}} \\
-k\left[\frac{\tan \left(\theta+\varphi_{0}\right)}{n}+\frac{\sec ^{3}\left(\theta+\varphi_{0}\right)}{2 \ln ^{2}} \hat{y}+\frac{3 \sec ^{4}\left(\theta+\varphi_{0}\right) \tan \left(\theta+\varphi_{0}\right)}{8 l^{2} n^{3}} \hat{y}^{2}\right]\left(x-\gamma_{0}+x_{0}\right) \\
+\left[\frac{4 c_{1} l^{2} \tan \left(\theta+\varphi_{0}\right)+3 n c_{2} \sec ^{2}\left(\theta+\varphi_{0}\right)}{2 l^{2} n^{2}}\right. \\
\left.+\left(4 c_{1} l^{2}+3 n c_{2}\right)\left(\frac{\sec ^{3}\left(\theta+\varphi_{0}\right) \tan \left(\theta+\varphi_{0}\right)}{2 l^{3} n^{3}} \hat{y}+\frac{\sec ^{6}\left(\theta+\varphi_{0}\right)\left(1+3 \sin ^{2}\left(\theta+\varphi_{0}\right)\right)}{8 l^{4} n^{4}} \hat{y}^{2}\right)\right] \dot{\hat{y}} \\
-c_{1}\left(\frac{\tan \left(\theta+\varphi_{0}\right)}{n}+\frac{\sec ^{3}\left(\theta+\varphi_{0}\right)}{2 \ln ^{2}} \hat{y}+\frac{3 \sec ^{4}\left(\theta+\varphi_{0}\right) \tan \left(\theta+\varphi_{0}\right)}{8 l^{2} n^{3}} \hat{y}^{2}\right) \dot{\hat{x}}=-M \ddot{z}_{y}-M g .
\end{array}\right.
$$

From Eq. (4d), it is revealed that the stiffness of the platform provides the support for loading since some terms in Eq. (12a) satisfy the condition of Eq. (4c). Based on Eq. (4c) and Eq. (12a), we expand all terms in the dynamic equation and rearrange it as

$$
\left\{\begin{array}{l}
M \ddot{\hat{x}}+\left(k+k_{e}\right) x-k\left[\frac{\tan \left(\theta+\varphi_{0}\right)}{n} \hat{y}+\frac{\sec ^{3}\left(\theta+\varphi_{0}\right)}{4 n^{2}} \hat{y}^{2}+\frac{\sec ^{4}\left(\theta+\varphi_{0}\right) \tan \left(\theta+\varphi_{0}\right)}{8 l^{2} n^{3}} \hat{y}^{3}\right] \\
+\left(c_{x}+2 c_{1}\right) \dot{\hat{x}}-c_{1}\left[\frac{\tan \left(\theta+\varphi_{0}\right)}{n}+\frac{\sec ^{3}\left(\theta+\varphi_{0}\right)}{2 n^{2}} \hat{y}+\frac{3 \sec ^{4}\left(\theta+\varphi_{0}\right) \tan \left(\theta+\varphi_{0}\right)}{8 l^{2} n^{3}} \hat{y}^{2}\right] \dot{\hat{y}}=-M \ddot{z}_{x}, \\
M \ddot{\hat{y}}+k\left[\frac{\tan ^{2}\left(\theta+\varphi_{0}\right)}{n^{2}} \hat{y}+\frac{3 \sec ^{3}\left(\theta+\varphi_{0}\right) \tan \left(\theta+\varphi_{0}\right)}{4 n^{3}} \hat{y}^{2}+\frac{\left(3-2 \cos 2\left(\theta+\varphi_{0}\right)\right) \sec ^{6} \theta}{8 l^{2} n^{4}} \hat{y}^{3}\right]+c_{y} \dot{\hat{y}} \\
-k \frac{\tan \left(\theta+\varphi_{0}\right)}{n} \hat{x}-k\left[\frac{\sec ^{3}\left(\theta+\varphi_{0}\right)}{2 \ln ^{2}} \hat{y}+\frac{3 \sec ^{4}\left(\theta+\varphi_{0}\right) \tan \left(\theta+\varphi_{0}\right)}{8 l^{2} n^{3}} \hat{y}^{2}\right]\left(\hat{x}-\frac{n M g}{k \tan \left(\theta+\varphi_{0}\right)}\right) \\
+\left[\frac{4 c_{1} l^{2} \tan ^{2}\left(\theta+\varphi_{0}\right)+3 n c_{2} \sec ^{2}\left(\theta+\varphi_{0}\right)}{2 l^{2} n^{2}}\right. \\
\left.+\left(4 c_{1} l^{2}+3 n c_{2}\right)\left(\frac{\sec ^{3}\left(\theta+\varphi_{0}\right) \tan \left(\theta+\varphi_{0}\right)}{2 l^{3} n^{3}} \hat{y}+\frac{\sec ^{6}\left(\theta+\varphi_{0}\right)\left(1+3 \sin ^{2}\left(\theta+\varphi_{0}\right)\right)}{8 l^{4} n^{4}} \hat{y}^{2}\right)\right] \dot{\hat{y}} \\
-c_{1}\left(\frac{\tan ^{2}\left(\theta+\varphi_{0}\right)}{n}+\frac{\sec ^{3}\left(\theta+\varphi_{0}\right)}{2 \ln ^{2}} \hat{y}+\frac{3 \sec ^{4}\left(\theta+\varphi_{0}\right) \tan \left(\theta+\varphi_{0}\right)}{8 l^{2} n^{3}} \hat{y}^{2}\right) \dot{\hat{x}}^{2}=-M \ddot{z}_{y} .
\end{array}\right.
$$

From (12b), it can be seen that the stiffness and damping properties of the 2-D SLS-based 
platform are nonlinear functions of displacement motion $x, y$ and velocity $\dot{x}, \dot{y}$. The responses subject to 2-D excitations are determined by the structural parameters $M, k, k_{e}, c_{x}, c_{y}, c_{1}, c_{2}, n, l, \theta$. Usually, the parameters $M, k, k_{e}, c_{x}, c_{y}, c_{1}$ and $c_{2}$ are fixed when the isolation object, springs, dampers and bearings are assembled while some structural parameters $n, l$ and $\theta$ in the SLS could be changed for better nonlinear stiffness and damping properties to satisfy engineering requirements. Therefore, the vibration isolation performance of the 2-D SLS-based platform would be studied with respect to each structural parameters $n, l$ and $\theta$ for understanding of parametric influence.

\section{Isolation performance of the 2-D SLS-based platform}

Note that the stiffness and damping of this system both converge to bounded real valued functions and can be approximated by Taylor series in a neighborhood of the equilibrium as discussed. The Harmonic Balance Method (HBM) is adopted to estimate the response of the 2-D nonlinear vibration isolation system in $(12)[42,44]$. If the excitations in the horizontal and vertical directions are considered as periodic signals, i.e., $z_{x}=z_{x 0} \cos (\omega t)$ and $z_{y}=z_{y 0} \cos (\omega t)$, the zero and the first order harmonic solutions of the relative motion $\hat{x}$ and $\hat{y}$ are

$$
\left\{\begin{array}{l}
\hat{x}(t)=a_{0}+a_{1} \cos \left(\omega t+\phi_{1}\right) \\
\hat{y}(t)=b_{0}+b_{1} \cos \left(\omega t+\phi_{2}\right)
\end{array}\right.
$$

where $a_{0}$ and $b_{0}$ are the offset of the vibration motion, $a_{1}$ and $b_{1}$ are amplitudes of the $1^{\text {st }}$ order harmonic response of the system, and $\phi_{1}$ and $\phi_{2}$ are phases. It can be checked that a second order or higher order harmonic solution gives the similar accuracy to the output response of the system as the first order harmonic solution (see also some discussions in [43]). The absolute motion $x$ and $y$ of the 2-D platform can be expressed as 


$$
\left\{\begin{aligned}
x(t)=\hat{x}(t)+z_{x}(t) & =a_{0}+a_{1} \cos \left(\omega t+\phi_{1}\right)+z_{x 0} \cos (\omega t) \\
& =a_{0}+\left(a_{1} \cos \phi_{1}+z_{x 0}\right) \cos \omega t-a_{1} \sin \phi_{1} \sin \omega t=a_{0}+A \cos \left(\omega t+\eta_{1}\right) \\
y(t)=\hat{y}(t)+z_{y}(t) & =b_{0}+b_{1} \cos \left(\omega t+\phi_{2}\right)+z_{y 0} \cos (\omega t) \\
& =b_{0}+\left(b_{1} \cos \phi_{2}+z_{y 0}\right) \cos \omega t-b_{1} \sin \phi_{2} \sin \omega t=b_{0}+B \cos \left(\omega t+\eta_{2}\right)
\end{aligned}\right.
$$

where $A$ and $B$ are the amplitudes of the absolute motions $x$ and $y$ with phases $\eta_{1}$ and $\eta_{2}$, respectively. When the frequencies in the two directions are different, the expressions of the solutions are similar to Eq. (14) and the derivation process is also the same as before in principle. The amplitudes $A$ and $B$ of the platform are

$$
\left\{\begin{array}{l}
A=\sqrt{\left(a_{1} \cos \phi_{1}+z_{x 0}\right)^{2}+\left(a_{1} \sin \phi_{1}\right)^{2}}=\sqrt{a_{1}^{2}+2 a_{1} z_{x 0} \cos \phi_{1}+z_{x 0}^{2}} \\
B=\sqrt{\left(b_{1} \cos \phi_{2}+z_{y 0}\right)^{2}+\left(b_{1} \sin \phi_{2}\right)^{2}}=\sqrt{b_{1}^{2}+2 b_{1} z_{y 0} \cos \phi_{2}+z_{y 0}^{2}}
\end{array}\right.
$$

The displacement transmissibility in the horizontal and vertical directions $T_{x}$ and $T_{y}$ are defined as

$$
\left\{\begin{array}{l}
T_{x}=\frac{A}{z_{x 0}}=\frac{\sqrt{a_{1}^{2}+z_{x 0}^{2}+2 a_{1} z_{x 0} \cos \phi_{1}}}{z_{x 0}} \\
T_{y}=\frac{B}{z_{y 0}}=\frac{\sqrt{b_{1}^{2}+z_{y 0}^{2}+2 a_{1} z_{y 0} \cos \phi_{1}}}{z_{y 0}}
\end{array}\right.
$$

\subsection{Natural Frequencies}

Usually, it is difficult to reduce the displacement transmissibility less than 1 in the frequency range of $[0, \sqrt{2} \Omega]$ for a $1-D O F$ vibration isolator. The dynamic equations (12) indicate that the 2-D SLS-based platform is a 2-DOF vibration system and the linear parts in terms of $x$ and $y$ are coupled. There will be two natural frequencies denoted by $\Omega_{1}$ and $\Omega_{2}$. It can be shown that there is an anti-resonance frequency band between the two natural frequencies where the displacement transmissibility is less than 1 .

Because the nonlinearity of the system is induced by nonlinear geometrical relations, it is difficult to exhibit bifurcation phenomenon if the excitation is at the centimeter level. From the 
linear parts of (12), the natural frequencies are dependent on five parameters i.e., $M, k, k_{e}, n$ and $\theta$. The parameters $M$ and $k$ are supposed to be fixed in a given practical engineering design, while $k_{e}, n$ and $\theta$ can be adjusted since the horizontal springs and the components in the SLS can be freely chosen. The values of natural frequencies of the system are then given by

$$
\begin{aligned}
\left|\mathbf{K}-\Omega^{2} \mathbf{M}\right| & =\left|\begin{array}{cc}
k+k_{e}-M \Omega^{2} & -k \frac{\tan \theta}{n} \\
-k \frac{\tan \theta}{n} & k \frac{\tan ^{2} \theta}{n^{2}}-M \Omega^{2}
\end{array}\right| \\
& =M^{2} \Omega^{4}-\left(k+k_{e}+k \frac{\tan ^{2}}{n^{2}}\right) M \Omega^{2}+k k_{e} \frac{\tan ^{2}}{n^{2}}=0
\end{aligned}
$$

It can be obtained that

$$
\Omega_{1,2}=\sqrt{\frac{k+k_{e}+k \frac{\tan ^{2} \theta}{n^{2}} \pm \sqrt{\left(k+k_{e}+k \frac{\tan ^{2} \theta}{n^{2}}\right)^{2}-4 k k_{e} \frac{\tan ^{2} \theta}{n^{2}}}}{2 M}}
$$

According to Eq. (18), the natural frequencies $\Omega_{1}$ and $\Omega_{2}$ are shown in Fig. 3 for different structural parameters $k_{e}, n$ and $\theta$.

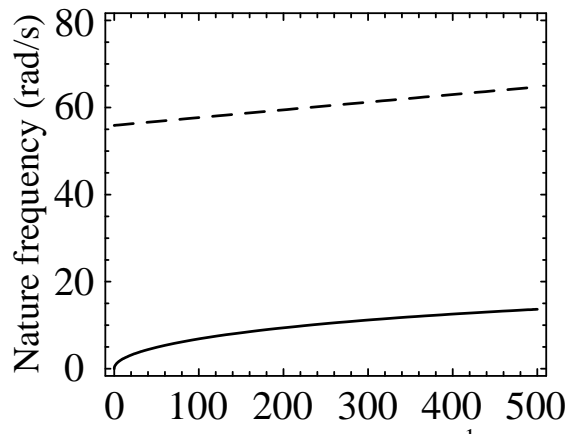

(a) Stiffness of $k_{e}\left(\mathrm{~N} \cdot \mathrm{m}^{-1}\right)$

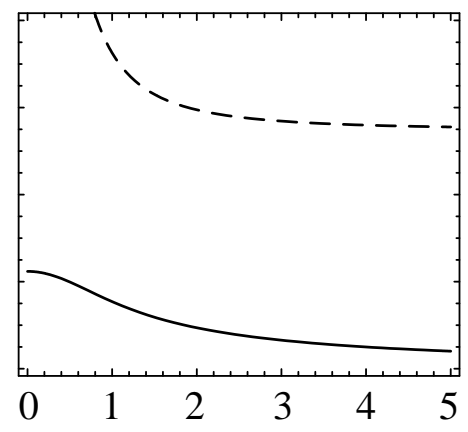

(b) Number of layer $n$

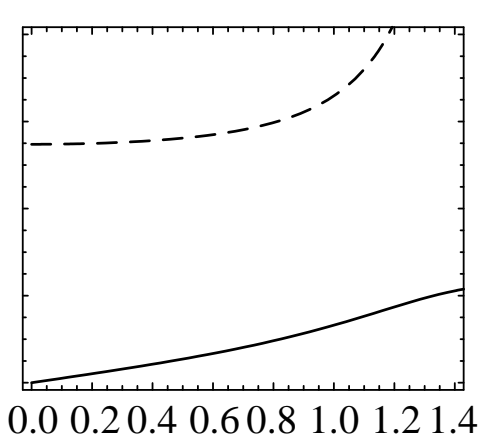

(c) Assembly angle $\theta(\mathrm{rad})$

Fig. 3 The value of first natural frequency $\Omega_{1}$ (solid lines) and second natural frequency $\Omega_{2}$ (dashed lines) for (a) different $k_{e}$; (b) different $n$; (c) different $\theta$.

From Fig. 3, it can be seen that the difference between the two natural frequencies $\Omega_{1}$ and $\Omega_{2}$ are dependent on the structural parameters. For smaller $k_{e}$ and $\theta$ or larger $n$, the anti-resonance frequency band is moving to low frequency and vice versa, indicating a tunable property with 
structure parameters. Low vibration transmissibility is expected to appear in the anti-resonance frequency band. Note also that, the values of $\Omega_{1}$ and $\Omega_{2}$ solved by linear parts of (12) are actually slightly affected by the nonlinear stiffness of the system. The influence of structural parameters $n, \theta$ and $l$ on the anti-resonance frequency band and its transmissibility are studied in the following parts.

\subsection{The isolation performance with respect to different structural parameters}

In the analysis above, larger $n$ or smaller $\theta$ can increase the anti-resonance frequency band and reduce the fundamental natural frequency of the 2-D isolation system while the value of $l$ has no effect on the value of linear stiffness of the system. However, according to (12), the three structural parameters all affect the values of nonlinear stiffness and damping coefficients. With the structural parameter settings in Table 1, the displacement transmissibility $T_{x}$ and $T_{y}$ for different $n, \theta$ and $l$ are shown in Fig. 4.
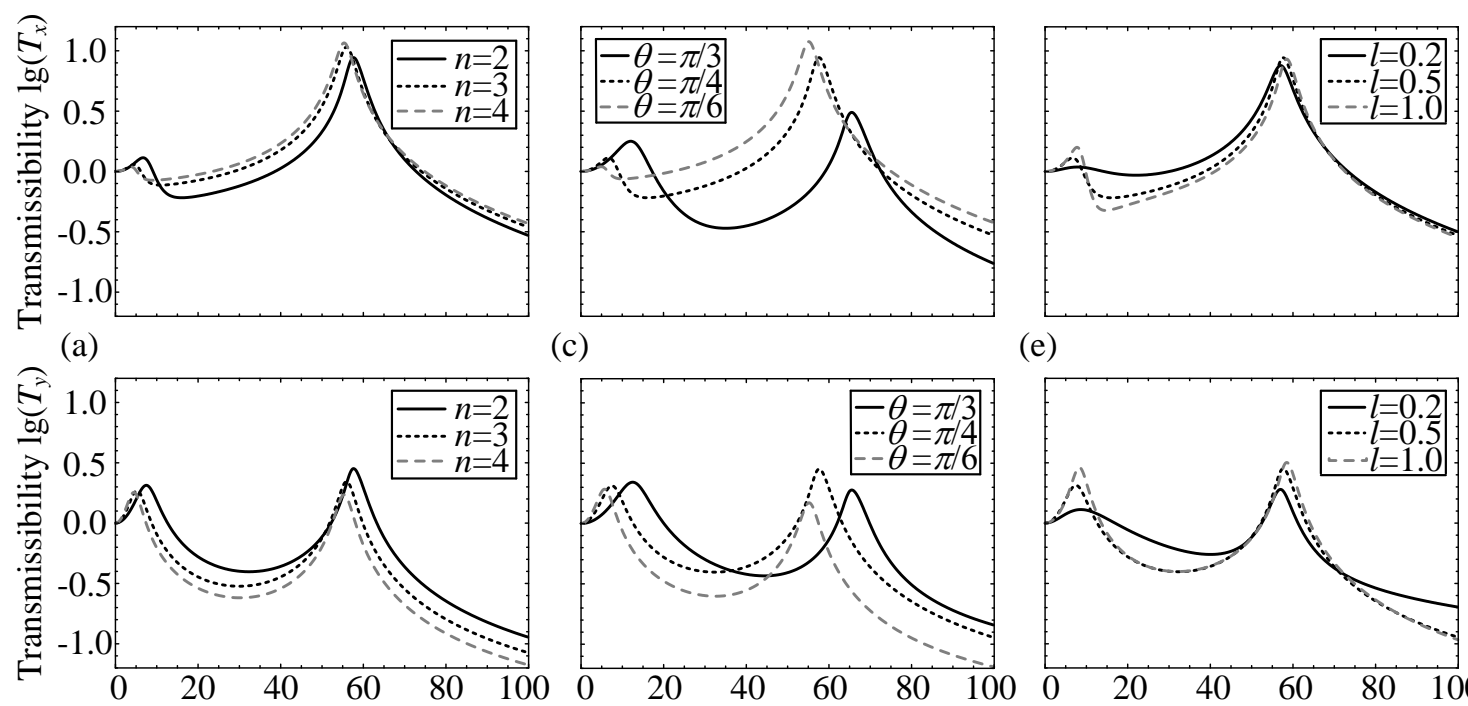

(c)

(e)

(b) Excitation frequency $\omega(\mathrm{rad} / \mathrm{s})$

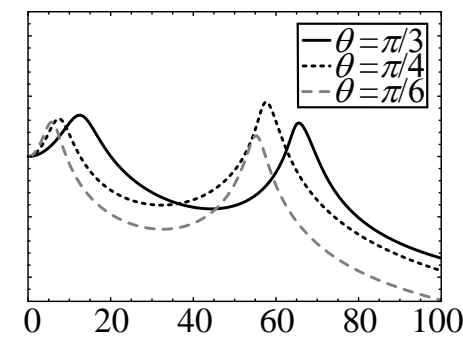

(d) Excitation frequency $\omega(\mathrm{rad} / \mathrm{s})$

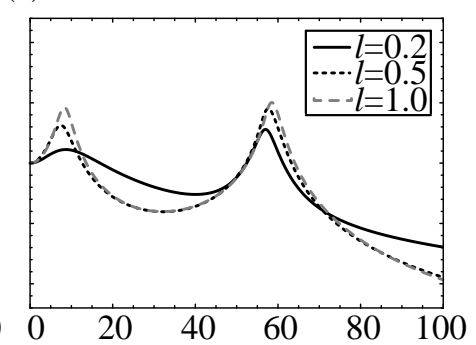

(f) Excitation frequency $\omega(\mathrm{rad} / \mathrm{s})$

Fig. 4 Displacement Transmissibilities $T_{x}$ in $(\mathrm{a}, \mathrm{c}, \mathrm{e})$ and $T_{y}$ in $(\mathrm{b}, \mathrm{d}, \mathrm{f})$ of the horizontal and vertical directions with different structural parameters.

Fig. 4 shows that the structural parameters $n, \theta$ and $l$ have obvious effect on the displacement 
transmissibility $T_{x}$ and $T_{y}$. As the value of $n$ increases, the isolation effect of $T_{x}$ is improved at the first resonance frequency while becomes worse at the anti-resonance frequency and the second resonance frequency; however, the isolation effect of $T_{y}$ is totally improved. Therefore, increasing the layer number of the SLS can improve the isolation effect in the vertical direction; but not always in the horizontal direction. From Fig. 4 (c) and (d), similar conclusions can be made for decreasing the value of $\theta$. From Fig. 4 (e) and (f), it can be seen that the effect of $l$ on the value of $T_{x}$ and $T_{y}$ is different from parameters $n$ and $\theta$. Firstly, changing the value of $l$ has little effect on the value of the two natural frequencies $\Omega_{1}$ and $\Omega_{2}$. Secondly, as the value of $l$ decreases, the displacement transmissibility is reduced at natural frequencies while it is increased in the anti-resonance frequency band because reducing the value of $l$ increases the damping coefficients of the system. Therefore, if the excitation frequency is in the anti-resonance band or at high frequency, the isolation effect in the horizontal and vertical directions can be improved by using longer connecting rods in the SLS platform. If the SLS-based platform is subject to random excitation, a smaller value of $l$ could be better.

From the analysis above, the influences of the three parameters $n, \theta$ and $l$ on the two-direction isolation performance are quite different, which are also very different from the conclusions in Ref. [36]. There should be a balance for the determination of $n$ and $\theta$ because too larger $n$ or smaller $\theta$ would induce worse isolation effect in the horizontal direction although these are helpful for the vertical direction isolation. Moreover, the vertical-direction vibration response can be reduced in the anti-resonance frequency band but increased at natural frequencies by increasing $l$.

\subsection{The effect of the friction coefficients $c_{1}$ and $c_{2}$}

To understand their influence, the displacement transmissibility $T_{x}$ and $T_{y}$ for different friction 
coefficients are shown in Fig. 5.

Increasing the friction coefficients $c_{1}$ and $c_{2}$ can reduce the resonant peak while have little effect on the transmissibility in the anti-resonance frequency band and at the low or high frequency in both directions. The latter is significantly different from the fact in pure linear vibration isolation systems that an increase of linear damping leads to the decrease of resonant peaks but increase of high-frequency transmissibility. This can be further seen in Section 4.1.

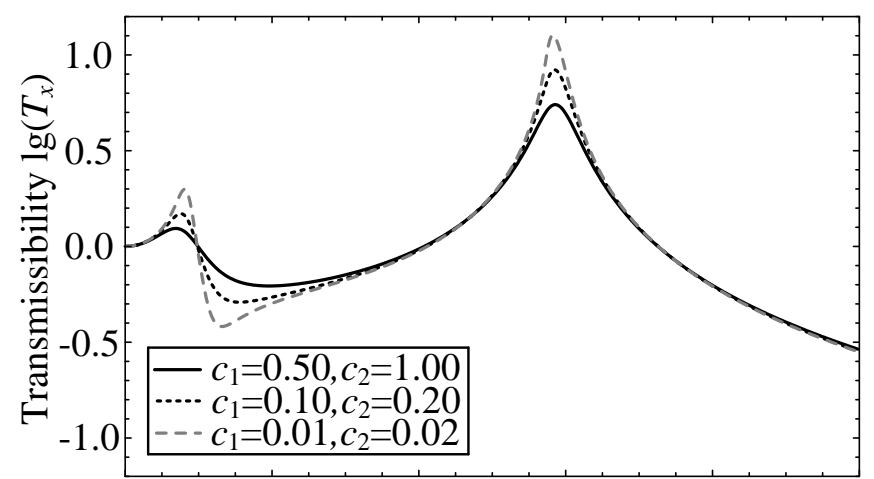

(a)

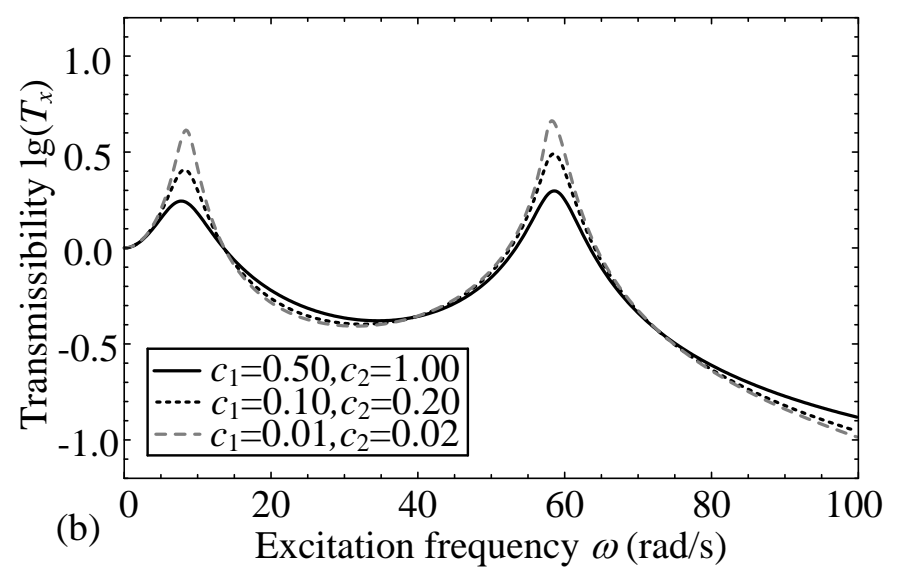

Fig. 5 Displacement transmissibilities $T_{x}$ in (a) and $T_{y}$ in (b) for different $c_{1}$ and $c_{2}$.

\section{Comparison studies}

\subsection{Compared with commonly-used mass-spring-damper systems}

Vibration isolation of a platform in different directions is usually done by applying springs in the corresponding directions (Fig. 6). It will be shown here that this commonly-used isolation 
method is not a good choice, which could be very worse than the proposed system.

\subsubsection{Modeling}

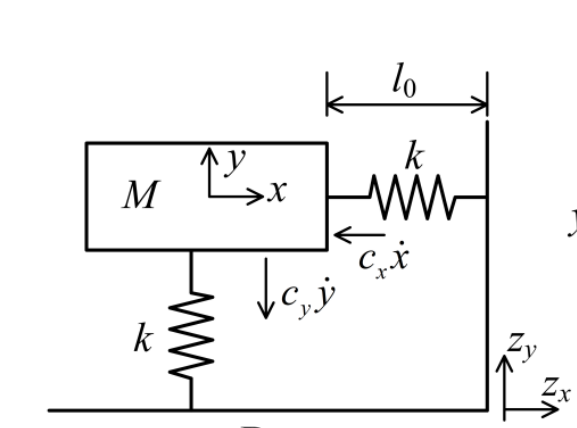

(a)

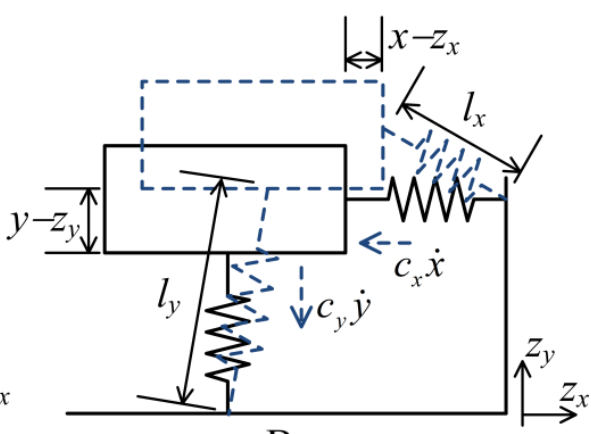

Base

(b)

Fig. 6 (a) A 2-D mass-spring-damper system. (b) Motions of the two springs in horizontal and vertical directions. Solid lines for original state and dashed lines for different vibration states.

For comparisons, consider a 2-D mass-spring-damper vibration isolator (MSD-VI) in Fig. 6. In Fig. 6 (a), the springs with the stiffness $k$ and original length $l_{0}$; the air-damping coefficients in the horizontal and vertical directions are $c_{x}$ and $c_{y}$, all the same as the SLS-based platform. The deformation of the horizontal spring $l_{x}$ could result in a deformation of the vertical spring $l_{y}$ and vice versa (Fig. 6 b). Therefore, in the modeling, the motion of the mass in one direction could affect its motion in the other direction, which induces the coupling nonlinearity. Firstly the static offset defined as $x_{0}$ and $y_{0}$ in two directions induced by the gravity should be obtained. Similar to the analysis of the SLS-VI, for static loading on the MSD platform, the gravitational energy of gravity part is $V_{g}=M g y_{0}$ and elastic potential energy induced by the gravity of loading is as $V_{e}=$ $\frac{1}{2} k\left(l_{0}-\sqrt{\left(l_{0}+x_{0}\right)^{2}+y_{0}^{2}}\right)^{2}+\frac{1}{2} k\left(l_{0}-\sqrt{\left(l_{0}+y_{0}\right)^{2}+x_{0}^{2}}\right)^{2}$. Based on the minimum potential energy principle, it has 


$$
\left\{\begin{array}{l}
\frac{\partial V_{e}}{\partial x_{0}}=-\frac{k\left(l_{0}+x_{0}\right)\left(l_{0}-\sqrt{\left(l_{0}+x_{0}\right)^{2}+y_{0}^{2}}\right)}{\sqrt{\left(l_{0}+x_{0}\right)^{2}+y_{0}^{2}}}-\frac{k x_{0}\left(-l_{0}+\sqrt{\left(l_{0}+y_{0}\right)^{2}+x_{0}^{2}}\right)}{\sqrt{\left(l_{0}+y_{0}\right)^{2}+x_{0}^{2}}}=0 \\
\frac{\partial V_{e}}{\partial y_{0}}+\frac{\partial V_{g}}{\partial y_{0}}=-\frac{k y_{0}\left(l_{0}-\sqrt{\left(l_{0}+x_{0}\right)^{2}+y_{0}^{2}}\right)}{\sqrt{\left(l_{0}+x_{0}\right)^{2}+y_{0}^{2}}}-\frac{k\left(l_{0}+y_{0}\right)\left(l_{0}-\sqrt{\left(l_{0}+y_{0}\right)^{2}+x_{0}^{2}}\right)}{\sqrt{\left(l_{0}+y_{0}\right)^{2}+x_{0}^{2}}}+M g=0
\end{array}\right.
$$

Expand Eq. (19a) by Taylor Series and it has

$$
\left\{\begin{array}{l}
k x_{0}+\frac{k x_{0} y_{0}}{l_{0}}+\frac{k x_{0}^{3}}{2 l_{0}^{2}}-\frac{k y_{0}^{2}}{2 l_{0}}-\frac{2 k x_{0} y_{0}^{2}}{l_{0}^{2}}=0 \\
k y_{0}+\frac{k x_{0}^{2}}{2 l_{0}}-\frac{k x_{0} y_{0}}{l}-\frac{2 k x_{0}^{2} y_{0}}{l_{0}^{2}}+\frac{k y_{0}^{3}}{2 l_{0}^{2}}=-M g
\end{array}\right.
$$

The kinetic energy $T$ of the MSD isolator is the same as (3) and the potential energy $V$ is dependent on the deformations of the vertical and horizontal springs which can be given by

$$
V=\frac{1}{2} k\left(l_{x}-l_{0}\right)^{2}+\frac{1}{2} k\left(l_{y}-l_{0}\right)^{2}+M g\left(y_{0}+y\right)
$$

The lengths $l_{x}$ and $l_{y}$ can be obtained by the geometrical relations according to Fig. 6, i.e., $l_{x}=\sqrt{\left(l_{0}-\hat{x}+x_{0}\right)^{2}+\left(\hat{y}+y_{0}\right)^{2}}$ and $l_{y}=\sqrt{\left(l_{0}+\hat{y}+y_{0}\right)^{2}+\left(\hat{x}+x_{0}\right)^{2}}$, where the static offset induced by gravity in the two direction is set as $x_{0}$ and $y_{0}$, relative motions are $\hat{x}=x-z_{x}$ and $\hat{y}=y-z_{y}$. Substituting $l_{x}$ and $l_{y}$ into the potential energy $V$ in (19) and applying the Langrage principle, the simplified dynamic equation of the MSD-VI after a similar Taylor Series expansion is

$$
\left\{\begin{array}{c}
M \ddot{\hat{x}}+k\left(\hat{x}+x_{0}\right)+\frac{k\left(\hat{x}+x_{0}\right)\left(\hat{y}+y_{0}\right)}{l_{0}}+\frac{k\left(\hat{x}+x_{0}\right)^{3}}{2 l_{0}^{2}}-\frac{k\left(\hat{y}+y_{0}\right)^{2}}{2 l_{0}} \\
-\frac{2 k\left(\hat{x}+x_{0}\right)\left(\hat{y}+y_{0}\right)^{2}}{l_{0}^{2}}+c_{x} \dot{\hat{x}}=-M \ddot{z}_{x} \\
M \ddot{\hat{y}}+k\left(\hat{y}+y_{0}\right)+\frac{k\left(\hat{x}+x_{0}\right)^{2}}{2 l_{0}}-\frac{k\left(\hat{x}+x_{0}\right)\left(\hat{y}+y_{0}\right)}{l}-\frac{2 k\left(\hat{x}+x_{0}\right)^{2}\left(\hat{y}+y_{0}\right)}{l_{0}^{2}} \\
+\frac{k\left(\hat{y}+y_{0}\right)^{3}}{2 l_{0}^{2}}+c_{y} \dot{\hat{x}}=-M \ddot{z}_{y}-M g
\end{array}\right.
$$

Because of Eq. (19b), the dynamic equation of the MSD-VI could be written as 


$$
\left\{\begin{array}{c}
M \ddot{\hat{x}}+k \hat{x}+\frac{k\left(\hat{x} \hat{y}+x_{0} \hat{y}+\hat{x} y_{0}\right)}{l_{0}}+\frac{k\left(\hat{x}^{3}+3 \hat{x}^{2} x_{0}+3 x_{0}^{2} \hat{x}\right)}{2 l_{0}^{2}}-\frac{k\left(\hat{y}^{2}+2 y_{0} \hat{y}\right)}{2 l_{0}} \\
-\frac{2 k\left(\hat{x}+x_{0}\right)\left(\hat{y}+y_{0}\right)^{2}}{l_{0}^{2}}+c_{x} \dot{\hat{x}}=-M \ddot{z}_{x} \\
M \ddot{\hat{y}}+k \hat{y}+\frac{k\left(\hat{x}^{2}+2 x_{0} \hat{x}\right)}{2 l_{0}}-\frac{k\left(\hat{x} \hat{y}+x_{0} \hat{y}+\hat{x} y_{0}\right)}{l}-\frac{2 k\left[\left(\hat{x}^{2}+2 x_{0} \hat{x}\right)\left(\hat{y}+y_{0}\right)+x_{0}^{2} \hat{y}\right]}{l_{0}^{2}} \\
+\frac{k\left(\hat{y}^{3}+3 y_{0}^{2} \hat{y}+3 y_{0} \hat{y}^{2}\right)^{3}}{2 l_{0}^{2}}+c_{y} \dot{\hat{x}}=-M \ddot{z}_{y}
\end{array}\right.
$$

Using the harmonic balance method, the responses in the two directions are the same as (15). Similarly to (16), the displacement transmissibility $T_{x}$ and $T_{y}$ are defined as $T_{x}=A / z_{x 0}$ and $T_{y}=B / z_{y 0}$.

\subsubsection{Performance Comparisons}

The first case is to achieve better isolation performance simultaneously in the horizontal and vertical directions. Taking $M=0.4, k=1000, c_{x}=2, c_{y}=2, z_{x 0}=0.02, z_{y 0}=0.02, l_{0}=1, k_{e}=200, c_{1}=0.1$, and $c_{2}=0.2$ for both systems (i.e., MSD and SLS), while changing $c_{x}=20$ and $c_{y}=20$ for the MSD and setting $n=2, \theta=\pi / 4$ and $l=0.5$ for the SLS, the displacement transmissibilities are shown in Fig. 7 .

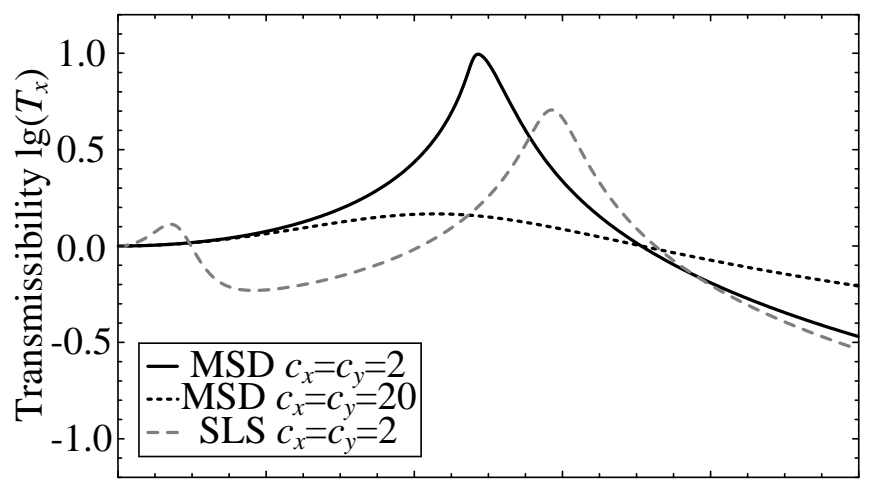

(a)

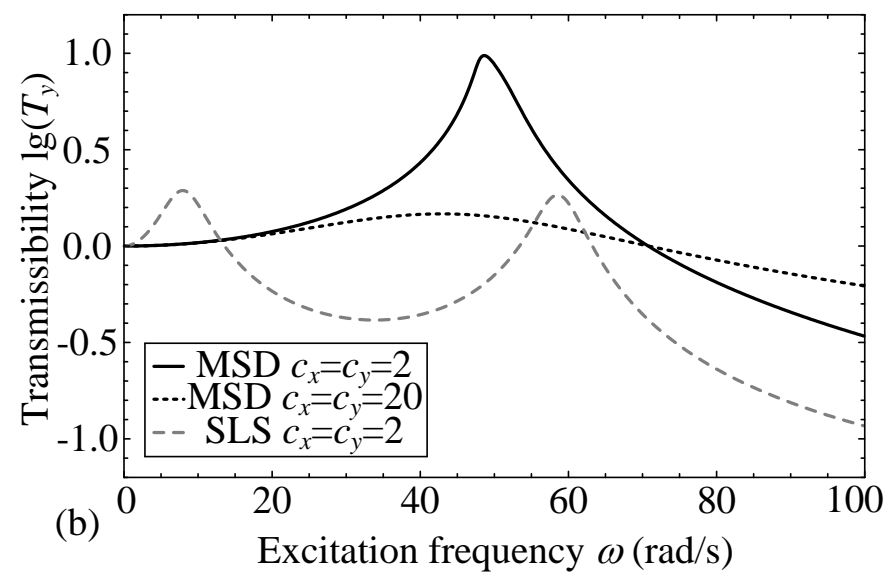


Fig. 7 Performance comparisons between the MSD-VI and SLS-based platform

In Fig. 7, although there is a resonant peak at low frequency for the SLS-based platform, the displacement transmissibility $T_{x}$ is reduced by about $50 \%$ and $T_{y}$ is reduced by about $80 \%$ at the second resonant peak, compared with the MSD system. For the MSD system, the resonant peak can be reduced by increasing the damping coefficients. However, the transmissibilities in the high and low frequency ranges are obviously increased which are not in the SLS-based platform. The displacement transmissibility $T_{x}$ and $T_{y}$ of the SLS-based platform can be reduced less than 1 in the anti-resonance frequency range, and most importantly, both can be designed to have a smaller resonant peak with simultaneously very low values at high frequencies. These cannot be obtained with the MSD system.

The second case study for comparisons is to partially sacrifice the isolation performance in the horizontal direction but to achieve much better vibration isolation in the vertical direction. Taking Taking $M=0.4, k=1000, c_{x}=2, c_{y}=2, z_{x 0}=0.02, z_{y 0}=0.02, l_{0}=1, k_{e}=200, c_{1}=0.1$, and $c_{2}=0.2$ for both systems, the displacement transmissibilities are shown in Fig. 8, where $n$ is increased to $n=3$ and $\theta$ is decreased to $\theta=\pi / 6$ for further improving the isolation effect of the SLS-based platform. 


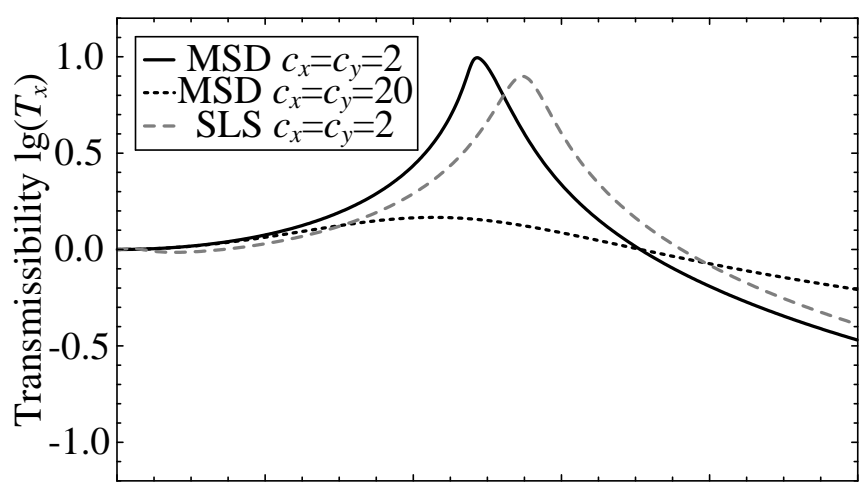

(a)

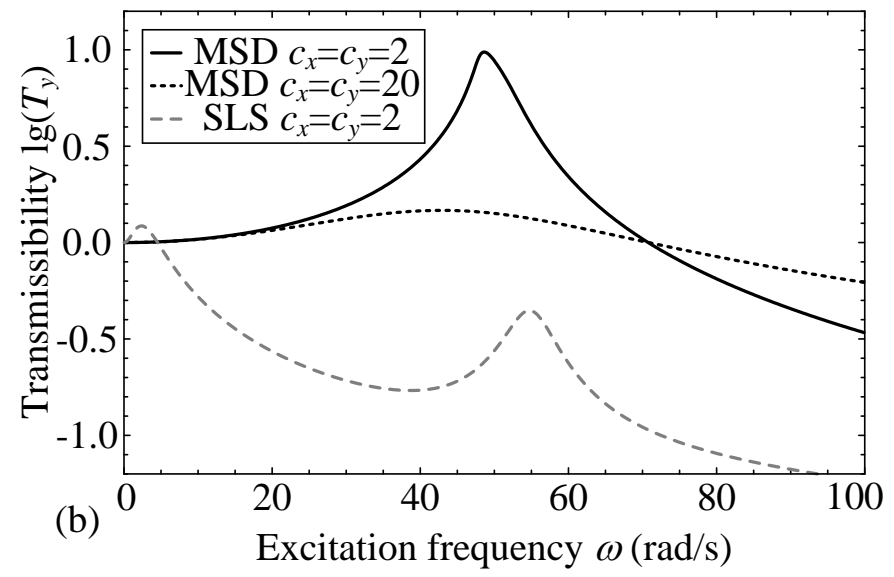

Fig. 8 Performance comparisons between the MSD-VI and SLS platform as $n=3, \theta=\pi / 6$ and $l=1$.

In Fig. 8, the displacement transmissibility $T_{y}$ of the SLS-based platform demonstrates obvious quasi-zero-stiffness property with significantly low transmissibility for all frequencies larger than about $1 \mathrm{rad} / \mathrm{s}$ and a very small resonant peak. This cannot be achieved by the passive MDS system with the same springs and mass. Although the isolation effect in the horizontal direction is worse than the first case, the SLS-based platform is still much better than the MSD system with the same linear damping coefficients.

\subsection{Compared with the existing quasi-zero-stiffness vibration isolator (QZS-VI)}

Where there is pre-compression in the horizontal springs in Fig 6, the system will become the QZS-VI studied in [6-15] which is a benchmark nonlinear vibration isolator which can achieve quasi-zero stiffness in the vertical direction (Fig 9). Note that the inherent nonlinearity of the QZS-VIs has an unstable equilibrium, easily leading to instability in practice, which is not in our 
proposed system. This section will show that our proposed system can still demonstrate better performance even though the unstable equilibrium of the QZS-VI is supposed not to appear under an ideal system design (no inherent nonlinearity in all springs, no unsymmetrical installment of horizontal springs, and ideal control of the pre-compression in the horizontal springs etc).

\subsubsection{Modeling}

The QZS-VI is shown in Fig. 9 (a) [6-15]. In the QZS system, the equivalent stiffness in the vertical direction can be reduced to a very small value by adjusting the pre-deformation of two horizontal springs. However, no result in the literature is focused on the isolation effect of the QZS-VI system in the horizontal direction.

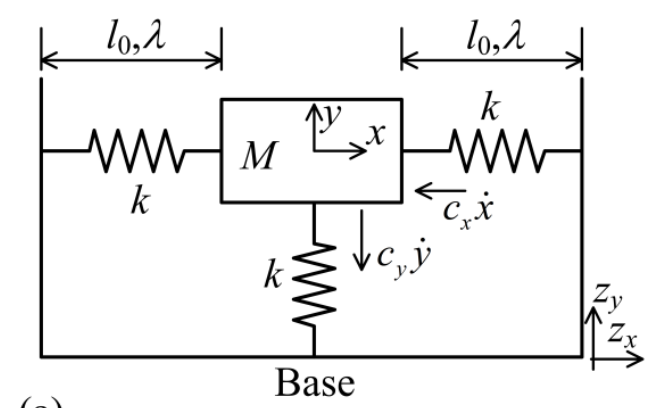

(a)

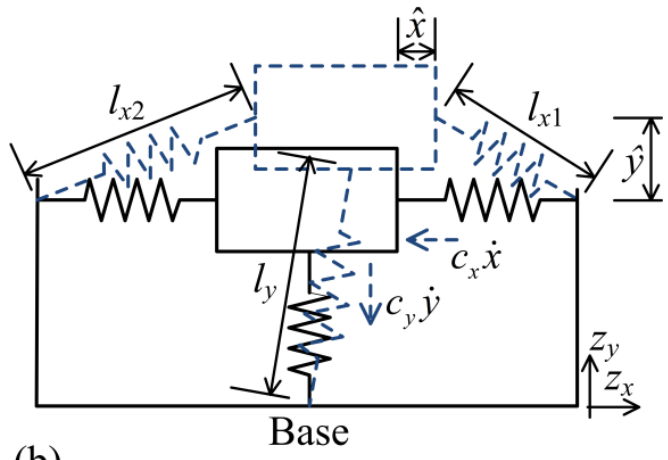

(b)

Fig. 9 (a) The QZS-VI system; (b) Deformations of the springs in the QZS-VI system. Solid lines for original state and dashed lines for different vibration positions.

The structural parameters in the QZS-VI system are set to the same as the SLS-based platform, i.e., the stiffness of horizontal springs and vertical spring is $k$, and the original length $l_{0}$. Moreover, the pre-deformation of horizontal springs in the QZS-VI is denoted by $\lambda$. For static loading on the QZS platform, the gravitational energy of gravity part is $V_{g}=M g y_{0}$ and elastic potential energy induced by the gravity of loading is as $V_{e}=\frac{1}{2} k\left(l_{0}-\sqrt{\left(l_{0}-\lambda+x_{0}\right)^{2}+y_{0}^{2}}\right)^{2}+\frac{1}{2} k\left(l_{0}-\right.$ $\left.\sqrt{\left(l_{0}-\lambda-x_{0}\right)^{2}+y_{0}^{2}}\right)^{2}+\frac{1}{2} k\left(l_{0}-\sqrt{\left(l_{0}+y_{0}\right)^{2}+x_{0}^{2}}\right)^{2}$. Based on the minimum potential energy principle, it has 


$$
\left\{\begin{array}{c}
\frac{\partial V_{e}}{\partial x_{0}}=\frac{k\left(l_{0}-\lambda-x_{0}\right)\left(l_{0}-\sqrt{\left(l_{0}-\lambda-x_{0}\right)^{2}+y_{0}^{2}}\right)}{\sqrt{\left(l_{0}-\lambda-x_{0}\right)^{2}+y_{0}^{2}}}-\frac{k\left(l_{0}-\lambda+x_{0}\right)\left(l_{0}-\sqrt{\left(l_{0}-\lambda+x_{0}\right)^{2}+y_{0}^{2}}\right)}{\sqrt{\left(l_{0}-\lambda+x_{0}\right)^{2}+y_{0}^{2}}} \\
-\frac{k x_{0}\left(l_{0}-\sqrt{\left(l_{0}+y_{0}\right)^{2}+x_{0}^{2}}\right)}{\sqrt{\left(l_{0}+y_{0}\right)^{2}+x_{0}^{2}}}=0 \\
\frac{\partial V_{e}}{\partial y_{0}}+\frac{\partial V_{g}}{\partial y_{0}}=-\frac{k y_{0}\left(l_{0}-\sqrt{\left(l_{0}-\lambda-x_{0}\right)^{2}+y_{0}^{2}}\right)}{\sqrt{\left(l_{0}-\lambda-x_{0}\right)^{2}+y_{0}^{2}}}-\frac{k y_{0}\left(l_{0}-\sqrt{\left(l_{0}-\lambda+x_{0}\right)^{2}+y_{0}^{2}}\right)}{\sqrt{\left(l_{0}-\lambda+x_{0}\right)^{2}+y_{0}^{2}}} \\
-\frac{k\left(l_{0}+y_{0}\right)\left(l_{0}-\sqrt{\left(l_{0}+y_{0}\right)^{2}+x_{0}^{2}}\right)}{\sqrt{\left(l_{0}+y_{0}\right)^{2}+x_{0}^{2}}}+M g=0
\end{array}\right.
$$

Expand Eq. (21a) by Taylor Series and it has

$$
\left\{\begin{array}{l}
k x_{0}+\frac{k x_{0}^{3}}{2 l_{0}^{2}}+\frac{k x_{0} y_{0}}{l_{0}}-\frac{k x_{0} y_{0}^{2}}{l_{0}^{2}}-\frac{2 k l_{0} x_{0} y_{0}^{2}}{\left(l_{0}-\lambda\right)^{3}}=0 \\
k \frac{l_{0}-3 \lambda}{l_{0}-\lambda} y_{0}+\frac{k l_{0}}{\left(l_{0}-\lambda\right)^{3}} y_{0}^{3}+\frac{k x_{0}^{2}}{2 l_{0}}-k x_{0}^{2} y_{0}\left[\frac{1}{l_{0}^{2}}+\frac{2 l_{0}}{\left(l_{0}-\lambda\right)^{3}}\right]=-M g
\end{array}\right.
$$

As shown in Fig. 9 (b), the lengths of the horizontal springs $l_{x 1}$ and $l_{x 2}$ and the length of the vertical spring $l_{y}$, have the following relations as

$$
\begin{aligned}
& l_{x 1}=\sqrt{\left(l_{0}-\lambda-\hat{x}-x_{0}\right)^{2}+\left(\hat{y}+y_{0}\right)^{2}}, \quad l_{x 2}=\sqrt{\left(l_{0}-\lambda+\hat{x}+x_{0}\right)^{2}+\left(\hat{y}+y_{0}\right)^{2}} \quad \text { and } \quad l_{y}= \\
& \sqrt{\left(l_{0}+\hat{y}+y_{0}\right)^{2}+\left(\hat{x}+x_{0}\right)^{2}} .
\end{aligned}
$$

The kinetic energy of the QZS-VI is the same as (3) and the potential energy $V$ is

$$
V=\frac{1}{2} k\left(l_{0}-l_{x 1}\right)^{2}+\frac{1}{2} k\left(l_{x 2}-l_{0}\right)^{2}+\frac{1}{2} k\left(l_{y}-l_{0}\right)^{2}+M g\left(y_{0}+y\right)
$$

Substituting $l_{x 1}, l_{x 2}$ and $l_{y}$ into the potential energy $V$, the dynamic equations of the QZS-VI in two-direction vibration could be obtained by Langrage principle similar as Refs. [8-15, 19-20]. For small excitation and response, the stiffness and damping of the system could be describe approximately by Taylor expansion, and then the dynamic equation of the QZS-VI is as 


$$
\left\{\begin{array}{l}
M \ddot{\hat{x}}+2 k\left(\hat{x}+x_{0}\right)+\frac{k\left(\hat{x}+x_{0}\right)\left(\hat{y}+y_{0}\right)}{l_{0}}+\frac{k\left(\hat{x}+x_{0}\right)^{3}}{2 l_{0}^{2}}-\left[\frac{k}{l_{0}^{2}}+\frac{2 k l_{0}}{\left(l_{0}-\lambda\right)^{3}}\right]\left(\hat{x}+x_{0}\right)\left(\hat{y}+y_{0}\right)^{2}+c_{x} \dot{\hat{x}}=-M \ddot{z}_{x} \\
M \ddot{\hat{y}}+k \frac{l_{0}-3 \lambda}{l_{0}-\lambda}\left(\hat{y}+y_{0}\right)+\frac{k\left(\hat{x}+x_{0}\right)^{2}}{2 l_{0}}-\left[\frac{k}{l_{0}^{2}}+\frac{2 k l_{0}}{\left(l_{0}-\lambda\right)^{3}}\right]\left(\hat{x}+x_{0}\right)^{2}\left(\hat{y}+y_{0}\right)+\frac{k l_{0}\left(\hat{y}+y_{0}\right)^{3}}{\left(l_{0}-\lambda\right)^{3}}+c_{y} \dot{\hat{x}}=-M \ddot{z}_{y}-M g
\end{array}\right.
$$

Based on Eq. (21b), the dynamic equation of the two-direction QZS-VI is as

$$
\left\{\begin{array}{c}
M \ddot{\hat{x}}+2 k \hat{x}+\frac{k\left(\hat{x} \hat{y}+x_{0} \hat{y}+\hat{x} y_{0}\right)}{l_{0}}+\frac{k\left(\hat{x}^{3}+3 \hat{x}^{2} x_{0}+3 \hat{x} x_{0}^{2}\right)}{2 l_{0}^{2}} \\
-\left[\frac{k}{l_{0}^{2}}+\frac{2 k l_{0}}{\left(l_{0}-\lambda\right)^{3}}\right]\left[\hat{x}\left(\hat{y}+y_{0}\right)^{2}+x_{0}\left(\hat{y}^{2}+2 \hat{y} y_{0}\right)\right]+c_{x} \dot{\hat{x}}=-M \ddot{z}_{x} \\
M \ddot{\hat{y}}+k \frac{l_{0}-3 \lambda}{l_{0}-\lambda} \hat{y}+\frac{k\left(\hat{x}^{2}+2 x_{0} \hat{x}\right)}{2 l_{0}}-\left[\frac{k}{l_{0}^{2}}+\frac{2 k l_{0}}{\left(l_{0}-\lambda\right)^{3}}\right]\left[\hat{y}\left(\hat{x}+x_{0}\right)^{2}+y_{0}\left(\hat{x}^{2}+2 \hat{x} x_{0}\right)\right] \\
+\frac{k l_{0}\left(\hat{y}^{3}+3 \hat{y}^{2} y_{0}+3 \hat{y} y_{0}^{2}\right)}{\left(l_{0}-\lambda\right)^{3}}+c_{y} \dot{\hat{x}}=-M \ddot{z}_{y}
\end{array}\right.
$$

From (22), increasing the value of pre-deformation $\lambda$ can reduce the value of linear stiffness coefficient, which is helpful for vibration isolation. However, the QZS-VI system should have enough stiffness to guarantee loading capacity and stability. The linear stiffness of the two directions should be positive value. From (22), it should satisfy $l_{0}-3 \lambda>0$. Therefore, the value of pre-deformation $\lambda$ should be in the range of $\left[0, l_{0} / 3\right](\mathrm{m})$ to keep a positive linear stiffness.

\subsubsection{Performance Comparison}

Considering the same stiffness of stiffness and damping coefficients in the QZS-VI and SLSbased platform as Table 1, the displacement transmissibility $T_{x}$ and $T_{y}$ are shown in Fig. 10. The resonant frequency of the QZS-VI is decreased by increasing the pre-deformation $\lambda$ of horizontal springs. For the stability of equilibrium, because of $l_{0}=1$, the value of pre-deformation $\lambda$ should satisfy $\lambda<l_{0} / 3 \approx 0.33$ to avoid bifurcation of equilibrium $[8,11]$. To investigate the improvement capacity of isolation effectiveness of the proposed SLS-based platform and the QZS-VI, the structural parameters of the SLS-based platform in Fig. 10 are set as the default values as $n=2$, 
$\theta=\pi / 4$ and $l=1$ and the pre-deformation of horizontal spring in QZS-VI is increased from 0.1 to 0.3 .

In Fig. 10, it can be seen that, the pre-deformation $\lambda$ of the horizontal springs has significant influence on the vibration isolation performance of the QZS-VI in the vertical direction but has little effect on the horizontal direction. The transmissibilities $\lg \left(T_{x}\right)$ and $\lg \left(T_{y}\right)$ of the SLS-based platform are smaller than those of the QZS-VI at around resonant frequencies, and both are less than 0 in anti-resonance frequency band. Different from the QZS-VI, adjusting the structural parameters $n, \theta$ and $l$ can improve the isolation effect in both vertical and horizontal directions and the vibration response in the vertical direction of the 2-D SLS-based platform can be reduced in a large frequency range without losing loading capacity.

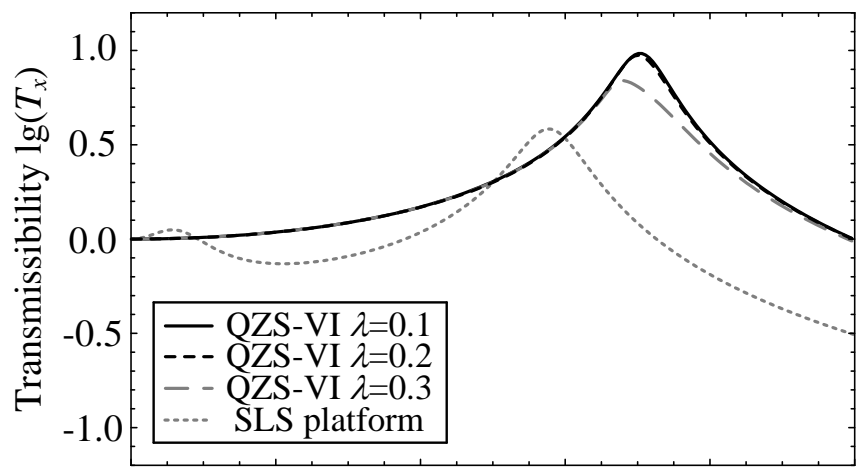

(a)

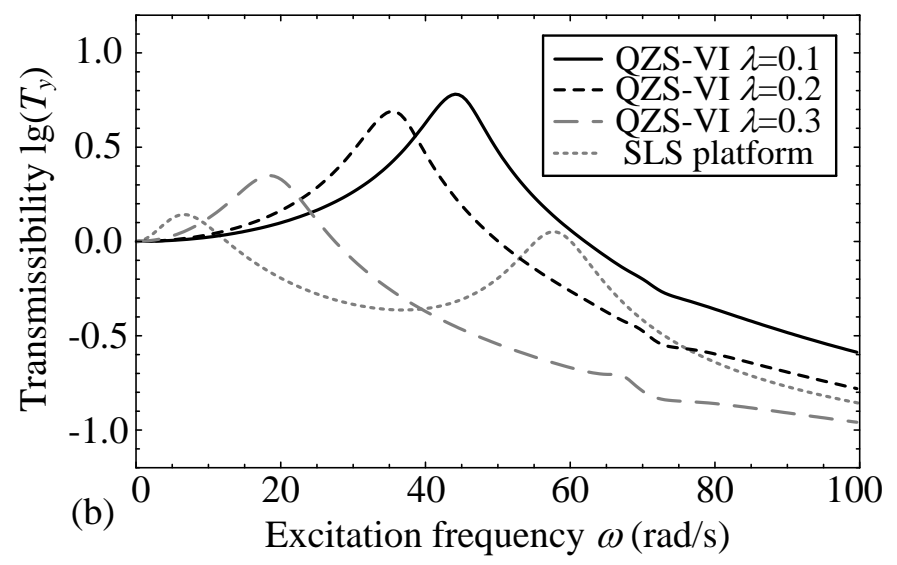

Fig. 10 Comparison between the QZS-VI and SLS platform. The overall performance of the proposed system is better or more preferable in practice both in the horizontal and vertical directions. 


\section{Case Study, Experiments and Discussions}

\subsection{Case Study}

Considering utilizing the proposed 2-D SLS platform as a vehicle seat suspension shown in Fig. 11, the isolation effect is studied, subjected to two-direction random base excitation and compared with the case utilizing linear active control in Ref. [34].

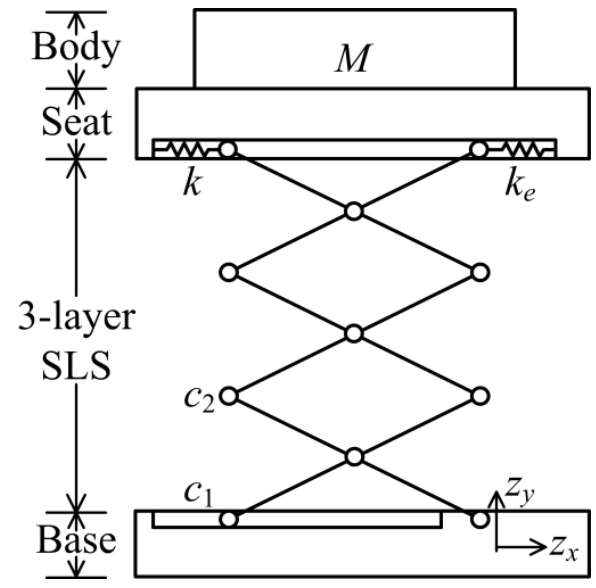

Fig. 11 Vehicle seat suspension model.

Because the vibration control system in [34] is only focused on vertical vibration isolation, the linear active control and excitation used there are applied in two orthogonal directions in order to show the advantages of the SLS platform in 2-D vibration isolation. The dynamics equation of the seat suspension system in [34] is given as

$$
\left\{\begin{array}{l}
M \ddot{\hat{x}}+c_{x} \dot{\hat{x}}+F_{x}(\hat{x})=-M \ddot{z}_{x} \\
M \ddot{\hat{y}}+c_{y} \dot{\hat{y}}+F_{y}(\hat{y})=-M \ddot{z}_{y}
\end{array}\right.
$$

where $F_{x}(\hat{x})$ and $F_{y}(\hat{y})$ are active control forces in $x$ and $y$ direction, respectively. The control laws are linear functions of relative motions as $F_{x}(\hat{x})=k \hat{x}$ and $F_{y}(\hat{y})=k \hat{y}$ where $k$ is the active control gain which can be treated as equivalent linear stiffness. The 2-D base excitations $z_{x}$ and $z_{y}$ are given as random signals which have frequency range from $1 \mathrm{~Hz}$ to $8 \mathrm{~Hz}$ and amplitude from $-0.02 \mathrm{~m}$ to 
$0.02 \mathrm{~m}$. For this random base excitation $z_{x}$ and $z_{y}$, the responses in $x$ and $y$ directions of the seat suspension with linear active control in (23) and the SLS platform are shown in Fig. 12. To simulate the cases as vehicle seat suspension, the corresponding parameters of the first case of the linear isolation system (23) are set as $M=100 \mathrm{~kg}, k=100000 \mathrm{~N} \cdot \mathrm{m}^{-1}, c_{x}=10, c_{y}=10$ and for improving the isolation effectiveness via active control, as the second case, the equivalent dynamic stiffness of the linear isolation system is reduced to $k=10000 \mathrm{~N} \cdot \mathrm{m}^{-1}$ and the equivalent damping coefficients are increased to $c_{x}=c_{y}=500$. For the SLS platform, the parameters are as same as the first case of the linear isolation system and additionally $k_{e}=2000 \mathrm{~N} \cdot \mathrm{m}^{-1}, n=3, \theta=\pi / 6$ and $l=0.2 \mathrm{~m}$.

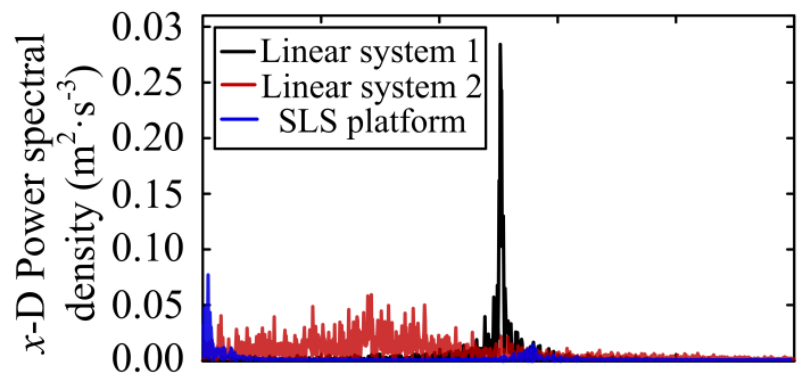

(a)

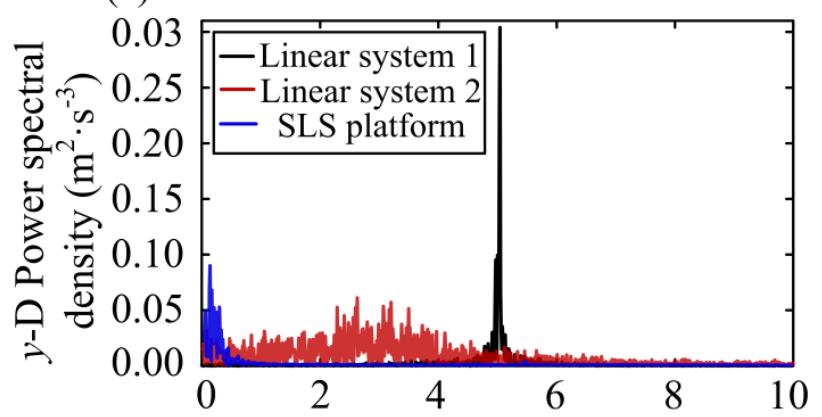

(c)
Excitation frequency $(\mathrm{Hz})$

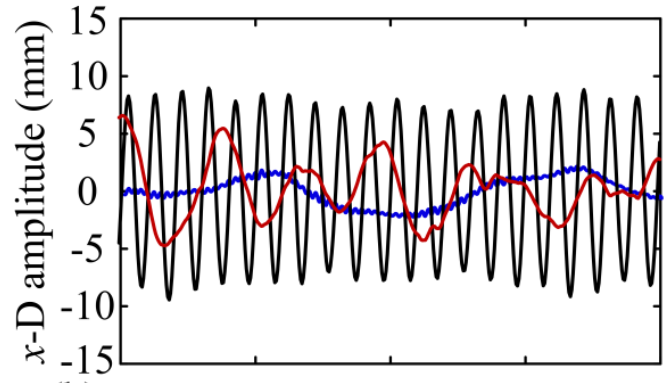

(b)

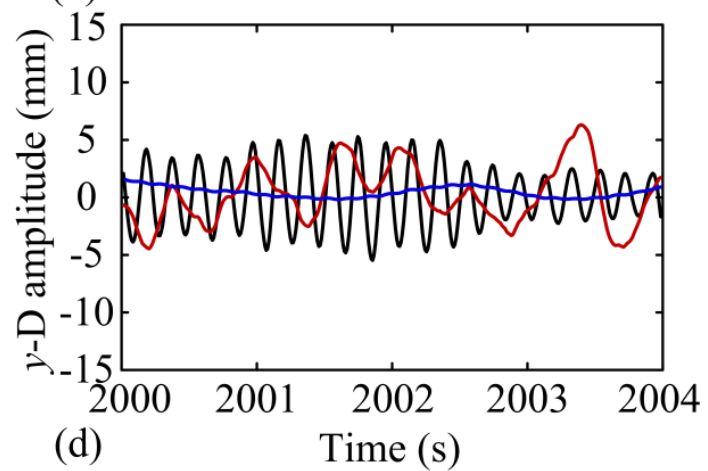

Fig. 12 Power spectral densities and time series of the seat suspension as [34] with linear active control device and the n-layer SLS platform for random base excitation; (a) Power spectral density in $x$ direction $(x-\mathrm{D})$ and (c) power spectral density in $y$ direction $(y$-D); (b) Time series in $x$ direction and (d) time series in $y$ direction.

For the first case of the linear isolation system as $M=100 \mathrm{~kg}$ and $k=100000 \mathrm{~N} \cdot \mathrm{m}^{-1}$, the natural frequency of the seat suspension system with active control is $5.033 \mathrm{~Hz}$ and the spectrum density 
has an obvious resonant peak. For the second case, as utilizing active control, the natural frequency is reduced to about $2 \mathrm{~Hz}$ and resonant peak is reduced much. While for the SLS platform, the resonant peak at critical frequency is greatly reduced, the first natural frequency is reduced to about $0.134 \mathrm{~Hz}$ and the second natural frequency is raised to $5.57 \mathrm{~Hz}$ because of the anti-resonance frequency band induced by the coupling linear parts of the two-direction vibration. Between the two natural frequencies, there is an anti-resonance frequency band where the amplitude is suppressed to very low.

\subsection{The experimental prototype}

The experimental prototype is simply designed to show the dominant quasi-zero-stiffness property in the vertical direction of the system in Fig 1. The prototype is shown in Fig. 13. When the stiffness of the horizontal spring at the top layer of the proposed 2-D SLS vibration platform in Fig. 1 is set to $k_{e} \rightarrow \infty$ (the motion of the right joint of the top layer can only rotate), the platform is then simplified to a one-direction vibration isolator as shown in Fig. 13. The spring horizontally assembled in the middle of the SLS in the prototype has equivalent effect as the spring $k$ assembled at the left side of the top layer of the SLS-based platform in Fig. 1. The stiffness of the linear spring using in the platform is $k(\cdot)$. The prototype has two layer SLS, i.e., $n=2$, with the assemble angle of the connecting rods $\theta=0.46 \mathrm{rad}$ and the rod length $l=0.2 \mathrm{~m}$. The spring stiffness is $k=1400 \mathrm{~N} \cdot \mathrm{m}^{-1}$ and the size of the upper platform is $0.2 \times 0.33 \times 0.003 \mathrm{~m}$. Because the material used is aluminum with density $2750 \mathrm{~kg} \cdot \mathrm{m}^{-3}$ and the mass of all accessories attached to the upper platform is about $0.18 \mathrm{~kg}$, the equivalent mass of the upper platform is about $M=0.18+2750 \times 0.2 \times 0.33 \times$ $0.003=0.7545 \mathrm{~kg}$. The resonant frequency of the system will become even smaller if the mass of the upper platform is increased. 


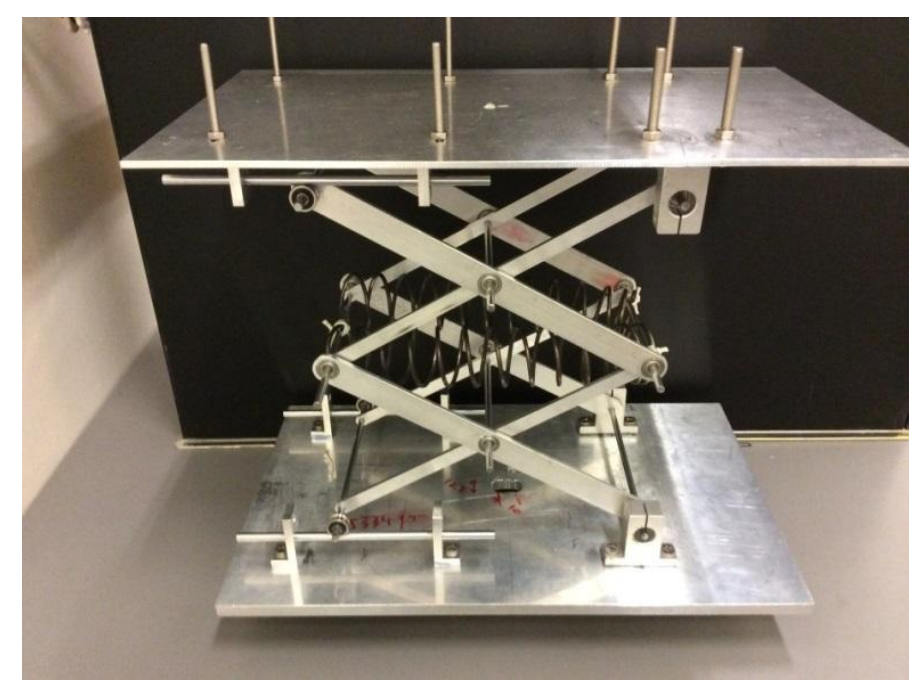

Fig. 13 The experimental prototype of the SLS-based platform

The weight of each connecting rod is only about $0.013 \mathrm{~kg}$ and the mass of each joint is much less than a rod. Therefore, the weights of rods and joints can be ignored in theoretical analysis. The damping of the horizontal tracks and joints can be considered as viscous functions because the bearings with proper lubrication can be well used.

According to Eq (20), a vertical 1-DOF MSD can be described by

$$
M \ddot{\hat{y}}+k_{s} \hat{y}+c_{s} \dot{\hat{y}}=M \ddot{z}_{y}
$$

For the prototype, considering the damping coefficients $c_{1}=c_{2}=c$ and only vertical vibration existing i.e., $x=z_{x}=0$ in (12), the dynamic equation of the prototype is

$$
\begin{aligned}
& M \ddot{\hat{y}}+k\left[\frac{\tan ^{2} \theta}{n^{2}} \hat{y}+\frac{3 \sec ^{3} \theta \tan \theta}{4 n^{3}} \hat{y}^{2}+\frac{(3-2 \cos 2 \theta) \sec ^{6} \theta}{8 l^{2} n^{4}} \hat{y}^{3}\right]+c_{y} \dot{\hat{y}} \\
& +c\left[\frac{4 l^{2} \tan ^{2} \theta+5 n \sec ^{2} \theta}{2 l^{2} n^{2}}+\left(4 l^{2}+5 n\right)\left(\frac{\sec ^{3} \theta \tan \theta}{2 l^{3} n^{3}} \hat{y}+\frac{\sec ^{6} \theta\left(1+3 \sin ^{2} \theta\right)}{8 l^{4} n^{4}} \hat{y}^{2}\right)\right] \dot{\hat{y}} \\
& =M \ddot{z}_{y}
\end{aligned}
$$

\subsection{Results and discussions}

\subsubsection{Natural frequency}

According to the linear stiffness coefficient in (25) of the platform, the theoretical natural 
frequency $\omega_{t}$ is

$$
\omega_{t}=\frac{\tan \theta}{n} \sqrt{\frac{k}{M}}=\frac{1}{4} \sqrt{\frac{1400}{0.7545}}=10.77 \quad\left(\mathrm{rad} \cdot \mathrm{s}^{-1}\right)=1.714 \quad(\mathrm{~Hz})
$$

To test the natural frequency of the platform, a random excitation with frequency from 0 to 100 $\mathrm{Hz}$ is used at the base and the vibration response of the upper platform is shown in Fig. 14.
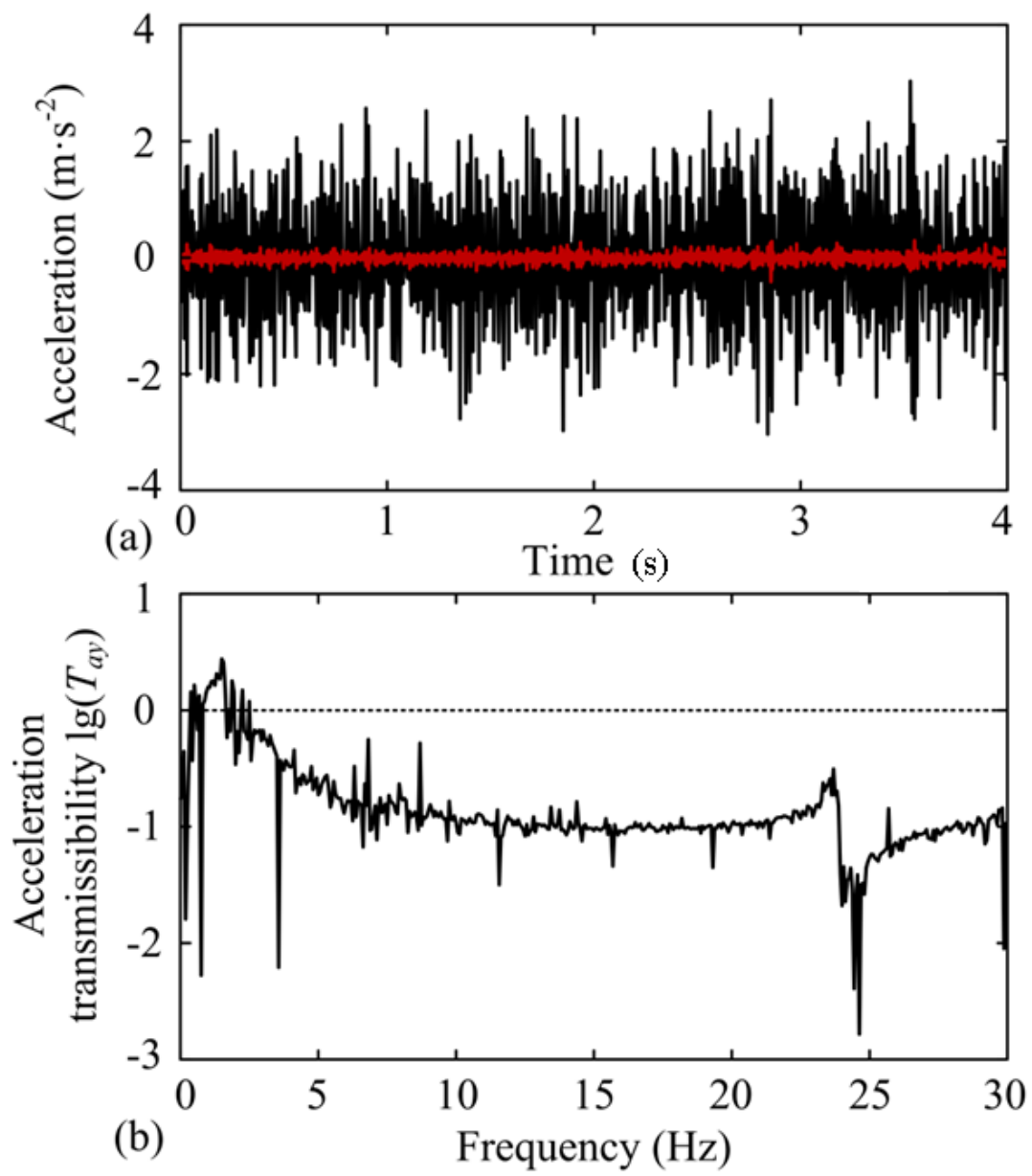

Fig. 14 Response and transmissibility of the platform for random excitation. (a) The time series accelerate signals of base excitation (Black) and platform (Red); (b) The acceleration transmissibility $T_{a y}$.

In Fig. 14 (b), the peak frequency of the acceleration transmissibility curve is about $1.6 \mathrm{~Hz}$, which is consistent with the theoretical analysis, i.e., $1.714 \mathrm{~Hz}$. The transmissibility in Fig. 14 (b) shows that the prototype has significant isolation effect for random base excitation and the 
transmissibility is much less than zero when the excitation frequency is larger than $2.5 \mathrm{~Hz}$. At higher frequencies larger than $5 \mathrm{~Hz}$, the transmissibility $T_{y}$ is roughly below $e^{-2}$ demonstrating very good isolation performance. From Fig. 14 (b), the second peak is at about $23 \mathrm{~Hz}$ which reflects the natural frequency for the flexibility of the connecting rods, and the displacement transmissibility at this peak is less than zero. Therefore, the flexibility of the connecting rods has little effect when the system is used as vibration isolator. If the mass of the upper platform is increased, the resonant frequency will become much smaller, which shows the quasi-zero stiffness property discussed before.

\subsubsection{Loading capacity}

The SLS platform has better static loading capacity than a traditional MSD isolator because of its nonlinear stiffness. Generally speaking, if the natural frequency of a traditional MSD isolator $\omega_{s}$ is designed to the same value as that of the SLS-based platform $\omega_{t}$ i.e., $\omega_{s}=\omega_{t}$, the stiffness of the MSD isolator can be obtained as

$$
k_{s}=\frac{\tan ^{2} \theta}{n^{2}} k
$$

Then the static deformation of the MSD isolator denoted by $\delta_{s}$ is

$$
\delta_{s}=\frac{M g}{k_{s}}=\frac{n^{2}}{\tan ^{2} \theta} \frac{M g}{k}
$$

Similarly, for this SLS platform, the static deformation $\delta$ of the platform can be obtained by the stiffness property in (23) as

$$
k\left[\frac{\tan ^{2} \theta}{n^{2}} \delta+\frac{3 \sec ^{4} \theta \sin \theta}{4 n^{3} l} \delta^{2}+\frac{(3-2 \cos 2 \theta) \sec ^{6} \theta}{8 n^{4} l^{2}} \delta^{3}\right]=M g
$$

which can be written as

$$
a \delta^{3}+b \delta^{2}+c \delta+d=0
$$


where $a=\frac{(3-2 \cos 2 \theta) \sec ^{6} \theta}{8 n^{4} l^{2}}, b=\frac{3 \sec ^{4} \theta \sin \theta}{4 n^{3} l}, c=\frac{\tan ^{2} \theta}{n^{2}}$ and $d=-\frac{M g}{k}$. The loading capacity of the proposed platform can be investigated from Eq. (30). Based on the condition for different kinds of roots for a cubic equation, there has only one real root when the structural parameters of the SLS platform and the loading $M$ are fixed in a specific range. For the cubic equation (30), when the coefficients satisfy the condition as $B^{2}-4 A C=(b c-9 a d)^{2}-4\left(b^{2}-3 a c\right)\left(c^{2}-3 b d\right)>0$, the equation has one real root and two complex roots. Therefore, it can be obtained that the static equilibrium of the SLS platform is unique and it is stable for load $M$ as

$$
27 M^{2} g^{2} n^{2}(3-2 \cos 2 \theta)^{2}+432 M g k n l \sin ^{5} \theta+4 k^{2} l^{2}(15-16 \cos 2 \theta) \sin ^{6} \theta>0
$$

when $15-16 \cos 2 \theta>0$, each term in Eq. (31) is positive, thus there is only one stable equilibrium as $\theta>0.177 \operatorname{rad} \approx 10.15^{\circ}$.

When a linear isolator has same resonant frequency as the proposed SLS platform which means that the stiffness of the linear isolator is $k_{S}=\frac{k \tan ^{2} \theta}{n^{2}}$, the static deformation for load $M$ is defined as $\delta_{s}=\frac{M g}{k \tan ^{2} \theta / n^{2}}$ as shown in Eq. (28). Then, Eq. (29) can be written as

$$
\delta+\frac{3 \sec ^{4} \theta \sin \theta}{4 n l \tan ^{2} \theta} \delta^{2}+\frac{(3-2 \cos 2 \theta) \sec ^{6} \theta}{8 n^{2} l^{2} \tan ^{2} \theta} \delta^{3}=\frac{n^{2}}{\tan ^{2} \theta} \frac{M g}{k}=\delta_{s}
$$

Eq. (32) shows that the static deformation of the SLS platform must be smaller than a commonly used MSD isolator, i.e., $\delta<<\delta_{s}$ for the same loading mass. That is also to say, for the same loading capacity, the SLS platform can have much smaller natural frequency. This clearly indicates the high-static-low-dynamic stiffness property of the SLS-based system. For the prototype with $k=1400 \mathrm{~N} \cdot \mathrm{m}^{-1}, n=2$ and $l=0.2 \mathrm{~m}$, the static deformations of the MSD isolator and the SLS platform for different assemble angle $\theta$ and loading mass $M$ are compared in Fig. 15 with $\omega_{i}=\omega_{s}$. 


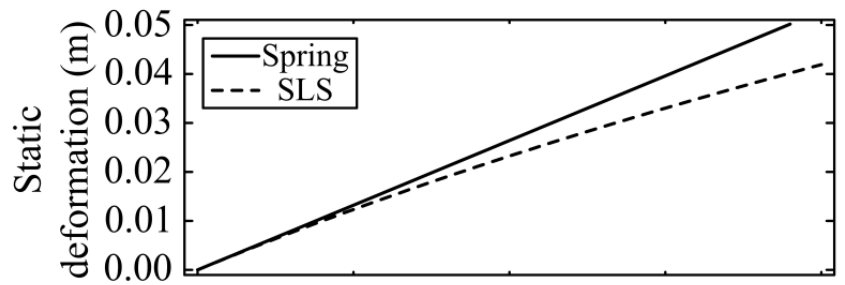

(a)

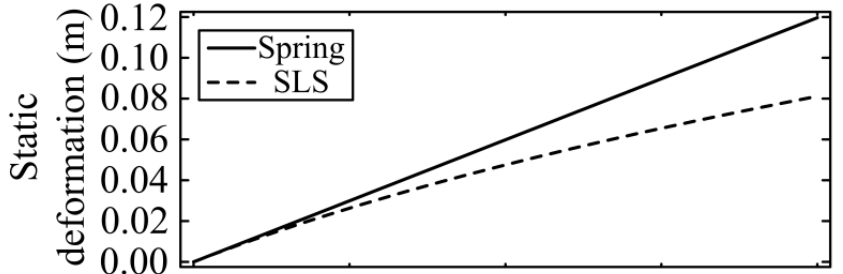

(b)

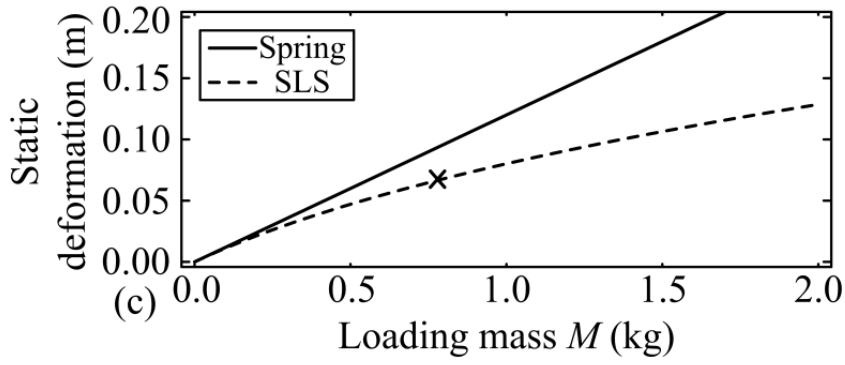

Fig. 15 The static deformation for different loading mass $M$ for (a) $\theta=0.8 \mathrm{rad}$, (b) $\theta=0.6 \mathrm{rad}$, and (c) $\theta=0.45 \mathrm{rad}$. The cross in (c) is the static deformation of the prototype

The cross " $X$ " in Fig. 15 (c) presents the experiment case conducted above. As shown in Fig. 15, for the SLS-based platform, with the increasing of the loading mass $M$, the increasing rate of the static deformation is much smaller because of the nonlinear stiffness. The nonlinear stiffness coefficients of the proposed isolation platform with the SLS are all positive, thus the isolation platform is deformed for a load $M$ and the equilibrium stability can be guaranteed when the static deformation is in a allowable range decided by the rod length $l$ and assembly angle $\theta$.

\subsubsection{Nonlinear damping performance}

Two responses of the SLS-based platform with only initial displacement excitations are shown in Fig. 16. In the tests, the upper platform is released from rest with a small positive or negative initial displacement. For the two tests, the responses both have a reasonable convergence rate with 6 periods in about 4 seconds. If a simple MSD isolator is used to reach similar response, the damping 
coefficient $c_{s}$ should satisfy

$$
c_{s}=2 \xi_{s} M \omega_{s}=2 \frac{1}{2 \pi \cdot 7} \ln \left(\frac{x_{0}}{x_{7}}\right) \cdot \sqrt{M k_{s}}=2 \frac{1}{2 \pi \cdot 7} \ln \left(\frac{250}{50}\right) \cdot \sqrt{66.02}=0.595 \mathrm{~N} \cdot \mathrm{m} \cdot \mathrm{s}^{-1}
$$

While for the SLS platform whose structural parameters are chosen as $n=2, \theta=0.45$ and $l=0.2$, the linear damping coefficient of the SLS platform is $c \frac{5 n \sec ^{2} \theta+4 l^{2} \tan ^{2} \theta}{2 l^{2} n}=78 c$. If the same linear damping coefficient is chosen for the SLS platform as $0.595 \mathrm{~N} \cdot \mathrm{m} \cdot \mathrm{s}^{-1}$ as in (33), then the damping coefficient of each bearing and horizontal track will be $c_{1}=c_{2}=c=0.0076 \mathrm{~N} \cdot \mathrm{m} \cdot \mathrm{s}^{-1}$. With this parameter setting, the transmissibility of the MSD isolator and the SLS platform are compared in Fig. 17.
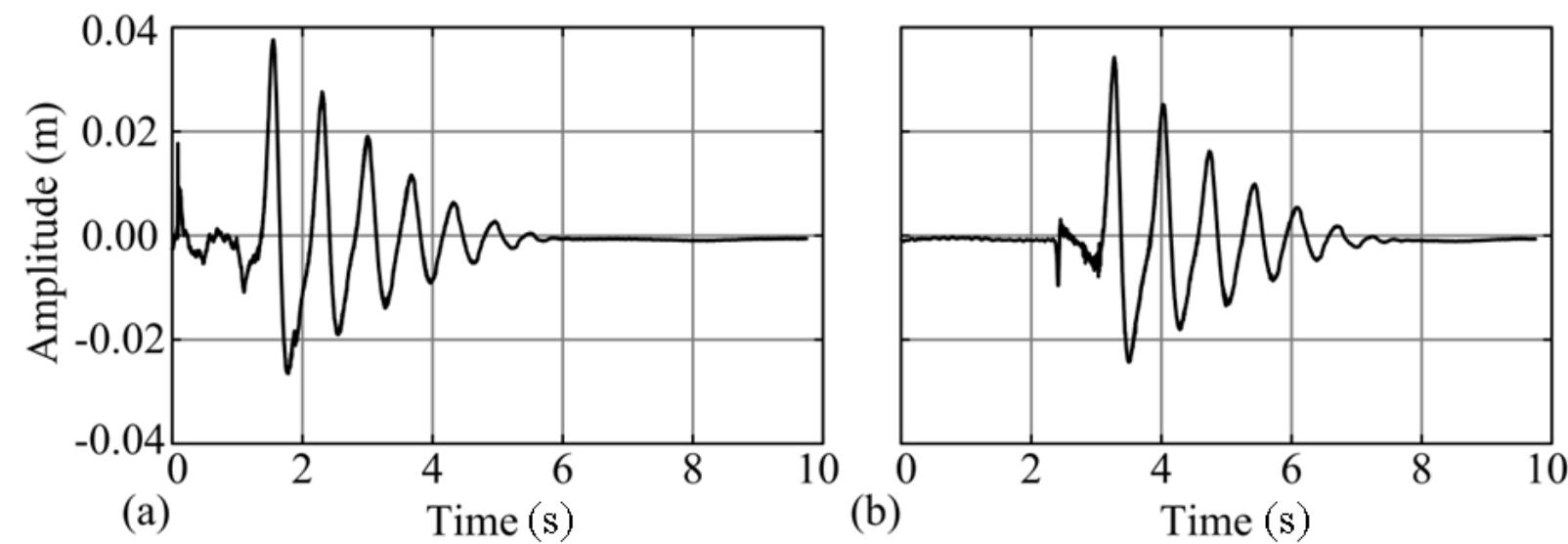

(b)

Fig. 16 Responses of the prototype with an initial displacement excitation for two times experiment.

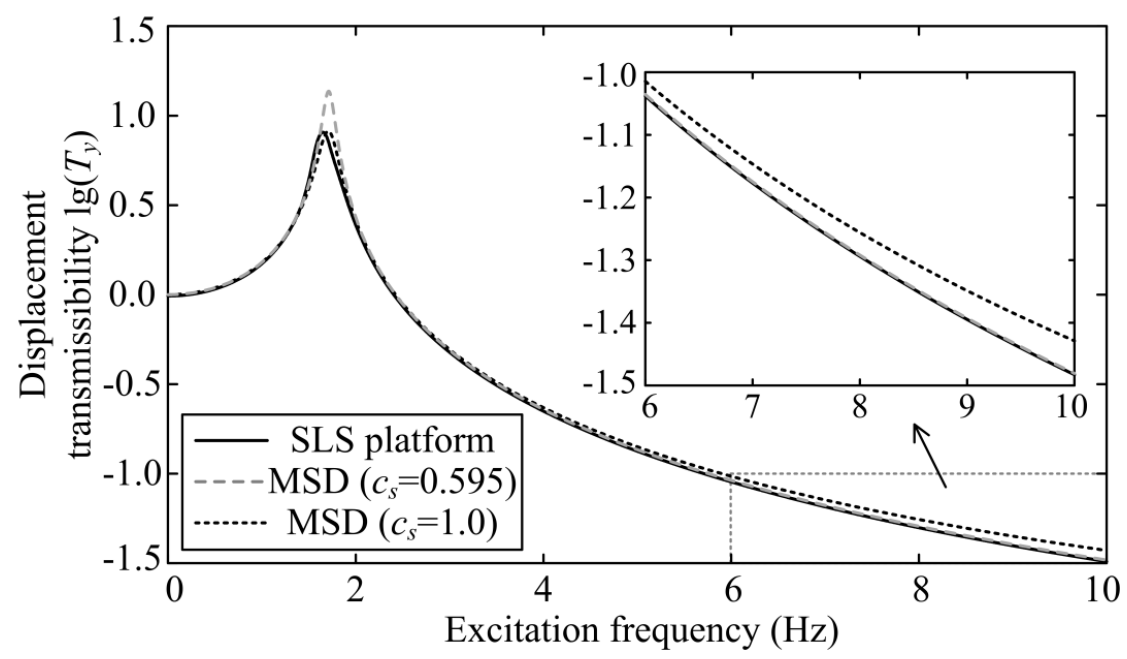

Fig. 17 Comparison between the MSD isolator and the SLS platform. 
It can be seen that the SLS platform has much smaller resonant peak but almost the same transmissibility at high frequencies as the MSD isolator with a smaller damping coefficient. To have a similar resonant peak by increasing linear damping, the MSD isolator will have much bigger transmissibility at high frequencies. This beneficial performance of nonlinear damping has been theoretically studied in [37-41]. The study here demonstrates a realistic, designable, and passive approach for realization of the advantageous nonlinear damping characteristics by using the SLS. This has not been concluded and practically demonstrated in previous studies [30-36].

\section{Conclusions}

This study proposed a novel n-layer 2-D SLS-based vibration isolation platform using only passive components. Theoretical analysis, comparison studies and experimental results demonstrate the advantages and versatility of the proposed system in vibration isolation because of nonlinearity. It can be concluded that,

(a) The proposed 2D SLS-based platform can achieve much better isolation performance (lower resonant frequency and peak values, stable equilibrium without jumping phenomenon, and robust to mass change) compared with existing QZS-VI system and traditional linear MSD isolator in two orthogonal directions, and the performance is designable with several structural parameters;

(b) Due to the coupling effect between the horizontal and vertical stiffness, the proposed system can achieve a very useful anti-resonance frequency band with tunable band frequency and magnitude via structural parameters, which is independent of the resonant frequency of the master system and is much better than the effect of traditional vibration 
absorbers;

(c) The scissor-like structural nonlinearity contributes both to nonlinear stiffness and nonlinear damping characteristics of the system, which are shown very beneficial in vibration control, leading to the high-static-low-dynamic-stiffness property and advantageous damping property (high damping at around resonant frequency but low at others).

The SLS can be flexibly constructed to achieve 3-D or above vibration isolation. Further studies will focus on prototyping of a specific practical application system and investigating the analysis and design of 3-D or 6-D vibration isolation systems using the scissor-like structure.

\section{Acknowledgment}

The authors would like to thank the editors and reviewers for their useful comments and constructive suggestions on this paper, and also gratefully acknowledge the support from a NSF project (Ref 61374041) of China, a GRF project (Ref 15206514) of Hong Kong RGC and internal research grants of Hong Kong Polytechnic University.

\section{Appendices}

Table 1 The structural parameters of the 2-D n-layer SLS-based platform and the default values in theoretical analysis.

\begin{tabular}{c|c|c|c}
\hline Symbol & Structural parameters & Unit & $\begin{array}{c}\text { Default } \\
\text { Value }\end{array}$ \\
\hline$k$ & linear stiffness & $\mathrm{N} \cdot \mathrm{m}^{-1}$ & 1000 \\
\hline$k_{e}$ & horizontal spring & $\mathrm{N} \cdot \mathrm{m}^{-1}$ & 200 \\
\hline$M$ & mass of isolation object & $\mathrm{kg}$ & 0.4 \\
\hline$n$ & number of layer & & 2 \\
\hline
\end{tabular}




\begin{tabular}{c|c|c|c}
\hline$l$ & length of connecting rod & $\mathrm{m}$ & 0.5 \\
\hline$\theta$ & assembly angle of connecting rod & $\mathrm{rad}$ & $\pi / 4$ \\
\hline$c_{x}$ & air damping coefficient in horizontal direction & $\mathrm{N} \cdot \mathrm{s} \cdot \mathrm{m}^{-1}$ & 2 \\
\hline$c_{y}$ & air damping coefficient in vertical direction & $\mathrm{N} \cdot \mathrm{s} \cdot \mathrm{m}^{-1}$ & 2 \\
\hline$c_{1}$ & friction coefficient of horizontal motion & $\mathrm{N} \cdot \mathrm{s} \cdot \mathrm{m}^{-1}$ & 0.1 \\
\hline$c_{2}$ & friction coefficient of rotational motion & $\mathrm{N} \cdot \mathrm{s} \cdot \mathrm{rad}^{-1}$ & 0.2 \\
\hline$F_{f x 1}$ & friction force of horizontal track in bottom layer & $\mathrm{N}$ & \\
\hline$F_{f x 2}$ & friction force of left horizontal track in top layer & $\mathrm{N}$ & \\
\hline$F_{f x 3}$ & friction force of right horizontal track in top layer & $\mathrm{N}$ & \\
\hline$F_{f \varphi}$ & friction force in $\varphi$ direction & $\mathrm{N}$ & \\
\hline$z_{x 0}$ & amplitude of horizontal excitation $z_{x}$ & $\mathrm{~m}$ & 0.02 \\
\hline$z_{y 0}$ & amplitude of vertical excitation $z_{y}$ & $\mathrm{~m}$ & 0.02 \\
\hline$\delta$ & static deformation & $\mathrm{m}$ & \\
\hline$\omega_{t}$ & natural frequency for $x=z_{x}=0$ & $\mathrm{rad} \cdot \mathrm{s}{ }^{-1}$ & \\
\hline
\end{tabular}

Table 2 The motion and variables for the SLS-based platform, MSD-VI and QZS-VI

\begin{tabular}{c|c|c}
\hline Symbol & Structural parameters & Unit \\
\hline$x_{0}$ & Horizontal offset of zero equilibrium induced by $M g$ & $\mathrm{~m}$ \\
\hline$y_{0}$ & Vertical offset of zero equilibrium induced by $M g$ & $\mathrm{~m}$ \\
\hline$x$ & absolute horizontal motion of isolation object & $\mathrm{m}$ \\
\hline$y$ & absolute vertical motion of isolation object & $\mathrm{m}$ \\
\hline$\hat{x}$ & relative motion in horizontal direction & $\mathrm{m}$ \\
\hline$\hat{y}$ & relative motion in vertical direction & $\mathrm{m}$ \\
\hline$\varphi$ & rotation transport motion & $\mathrm{rad}$ \\
\hline$\gamma_{x}$ & horizontal transport motion & $\mathrm{m}$ \\
\hline$z_{x}$ & horizontal excitation from base & $\mathrm{m}$ \\
\hline$z_{y}$ & vertical excitation from base & $\mathrm{m}$ \\
\hline$\omega$ & frequency of base excitation & $\mathrm{rad} \cdot \mathrm{s}^{-1}$ \\
\hline$\Omega_{1}$ & first order of natural frequency & $\mathrm{rad} \cdot \mathrm{s}^{-1}$ \\
\hline$\Omega_{2}$ & second order of natural frequency & $\mathrm{rad} \cdot \mathrm{s}^{-1}$ \\
\hline$T_{x}$ & displacement transmissibility in horizontal direction & \\
\hline$T_{y}$ & displacement transmissibility in vertical direction & \\
\hline & & \\
\hline
\end{tabular}

\section{References}

[1] E.I. Rivin, Vibration isolation of precision equipment. Precision Engineering 17 (1) (1995) 41-56. doi: 10.1016/0141-6359(94)00006-L 
[2] J. Yang, S.S. Sun, T.F. Tian, W.H. Li et.al, Development of a novel multi-layer MRE isolator for suppression of building vibrations under seismic events. Mechanical Systems and Signal Processing (In Press) (2015). doi:10.1016/j.ymssp.2015.08.022

[3] L.K. Liu, G.T. Zheng, W.H. Huang, Octo-strut vibration isolation platform and its application to whole spacecraft vibration isolation. Journal of Sound and Vibration 289 (4-5) (2006) 726-744. doi: 10.1016/j.jsv.2002.02.040

[4] K. Denoyer, C. Johnson, Recent achievements in vibration isolation systems for space launch and on-orbit applications, 52nd International Astronautical Congress, Toulouse, France, Oct. 2001, pp. 1-11.

[5] International Prganization for Standardization, ISO 2631-2, Mechanical vibration and shock-evolution of human exposure to whole-body vibration, 1997.

[6] R.A. Ibrahim, Recent advances in nonlinear passive vibration isolators. Journal of Sound and Vibration 314 (3-5) (2008) 371-452. doi: 10.1016/j.jsv.2008.01.014

[7] L.N. Virgin, S.T. Santillan, R.H. Plaut, Vibration isolation using extreme geometric nonlinearity. Journal of Sound and Vibration 314 (3) (2008) 721-731. doi: 10.1016/j.jsv.2007.12.025

[8] A. Carrella, M.J. Brennan, T.P. Waters, Static analysis of a passive vibration isolator with quasi-zero-stiffness characteristic. Journal of Sound and Vibration 301 (3-5) (2007) 678-689. doi: 10.1016/j.jsv.2006.10.011

[9] I. Kovacic, M.J. Brennan, T.P. Waters, A study of a nonlinear vibration isolator with a quasi-zero stiffness characteristic. Journal of Sound and Vibration 315 (3) (2008) 700-711. doi: 10.1016/j.jsv.2007.12.019

[10]A. Carrella, M.J. Brennan, I. Kovacic, T.P. Waters, On the force transmissibility of a vibration 
isolator with quasi-zero-stiffness. Journal of Sound and Vibration 322 (4-5) (2009) 707-717. doi: 10.1016/j.jsv.2008.11.034

[11] W.S. Robertson, M.R.F. Kidner, B.S. Cazzolato, A.C. Zander, Theoretical design parameters for a quasi-zero stiffness magnetic spring for vibration isolation. Journal of Sound and Vibration 326 (1-2) (2009) 88-103. doi: 10.1016/j.jsv.2009.04.015

[12]N. Zhou, K. Liu, A tunable high-static-low-dynamic stiffness vibration isolator. Journal of Sound and Vibration 329 (9) (2010) 1254-1273. doi: 10.1016/j.jsv.2009.11.001

[13]A. Carrella, M.J. Brennan, T.P. Waters, V. Lopes Jr., Force and displacement transmissibility of a nonlinear isolator with high-static-low-dynamic-stiffness. International Journal of Mechanical Sciences 55 (1) (2012) 22-29. doi: 10.1016/j.ijmecsci.2011.11.012

[14]X.T. Sun, X.J. Jing, Multi-direction vibration isolation with quasi-zero stiffness by employing geometrical nonlinearity. Mechanical Systems and Signal Processing 62-63 (2015) 149-163. doi:10.1016/j.ymssp.2015.01.026

[15]T.D. Le, K.K. Ahn, A vibration isolation system in low frequency excitation region using negative stiffness structure for vehicle seat. Journal of Sound and Vibration 330 (26) (2011) 6311-6335. doi: 10.1016/j.jsv.2011.07.039

[16]C.M. Lee, A.H. Bogatchenkov, V.N. Goverdovskiy, Y.V. Shynkarenko, A.I. Temnikov, Position control of seat suspension with minimum stiffness. Journal of Sound and Vibration 292 (1-2) (2006) 435-442. doi: 10.1016/j.jsv.2005.08.027

[17]C.M. Lee, V.N. Goverdovskiy, A multi-stage high-speed railroad vibration isolation system with "negative" stiffness. Journal of Sound and Vibration 331 (4) (2012) 914-921. doi: 10.1016/j.jsv.2011.09.014 
[18]G.D. Massa, R. Russo, S. Strano, M. Terzo, System structure identification and adaptive control of a seismic isolator test rig. Mechanical Systems and signal Processing 40 (2) (2013) 736-753. doi:10.1016/j.ymssp.2013.06.029

[19]R.L. Tian, Q.J. Cao, Z.X. Li, Hopf bifurcations for the recently proposed smooth-and-discontinuous oscillator. Chinese Physics Letters 27 (7) (2010) 074701.

[20] Y.W. Han, Q.J. Cao, Y.S. Chen, M. Wiercigrogh, A novel smooth and discontinuous oscillator with strong irrational nonlinearities. Science China: Physics, Mechanics and Astronomy 55 (10) (2012) 1832-1843.

[21]Q.J. Cao, Y.W. Han, T.W. Liang, M. Wiercigroch, S. Piskarev, Multiple buckling and codimension-three bifurcation phenomena of a nonlinear oscillator. International Journal of Bifurcation and Chaos 29 (24) (2014) 1430005.

[22]M. Sayed, M. Kamel, 1:2 and 1:3 internal resonance active absorber for non-linear vibrating system. Applied Mathematical Modelling 36 (1) (2011) 310-332. doi: 10.1016/j.apm.2011.05.057

[23]J.C. Ji, Design of a nonlinear vibration absorber using three-to-one internal resonances. Mechanical Systems and Signal Processing 42 (s 1-2) (2014) 236-246.

[24]F. Weber, Semi-active vibration absorber based on real-time controlled MR damper. Mechanical Systems and Signal Processing 46(2) (2014) 272-288.

[25]L.L. Sun, C.H. Hansen, C. Doolan, Evaluation of the performance of a passive-active vibration isolation system. Mechanical Systems and Signal Processing 50-51 (2015) 480-497. doi:10.1016/j.ymssp.2014.05.008

[26]G. Gatti, M.J. Brennan, I. Kovacic, On the interaction of the responses at the resonance 
frequencies of a nonlinear two degrees-of-freedom system. Physica D 239 (10) (2010) 591-599. doi: 10.1016/j.physd.2010.01.006

[27]S.J. Jang, M.J. Brennan, E. Rustighi, H.J. Jung, A simple method for choosing the parameters of a two degree-of-freedom tuned vibration absorber. Journal of Sound and Vibration 331 (21) (2012) 4658-4667. doi: 10.1016/j.jsv.2012.05.020

[28]J.C. Ji, N. Zhang, Suppression of the primary resonance vibrations of a forced nonlinear system using a dynamic vibration absorber. Journal of Sound and Vibration 329 (11) (2010) 2044-2056. doi: 10.1016/j.jsv.2009.12.020

[29]T. Detroux, G. Habib, L. Masset, G. Kerschen, Performance, robustness and sensitivity analysis of the nonlinear tuned vibration absorber. Mechanical Systems and Signal Processing s60-61 (2015) 799-809.

[30]A.N. Sigaher, M.C. Constantinou, Scissor-jack-damper energy dissipation system. Earthquake Spectra 19 (1) (2003) 133-158. doi:

[31]M.H. Rafieipour, M. Ziyaeifar, A.K. Ghorbani-Tanha, M. Rahimian, R. Mohammadi-Ghazi, Combination of an innovative instrument with a viscous damper to control the structure vibrations. World of Sciences Journal 1 (7) (2013) 141-162.

[32]C.M. Park, H.J. Jung, J.E. Jang, K.S. Park, I.W. Lee, Scissor-jack-damper system for reduction of cable vibration. The Eighteenth KKCNN Symposium on Civil Engineering-KAIST7, Taiwan (2005) 18-20.

[33]D. Fischer, R. Isermann, Mechatronic semi-active and active vehicle suspensions. Control Engineering Practice 12 (11) (2004) 1353-1367. doi: 10.1016/j.conengprac.2003.08.003

[34]I. Maciejewski, L. Meyer, T. Krzyzynski, The vibration damping effectiveness of an active seat 
suspension system and its robustness to varying mass loading. Journal of Sound and Vibration 329 (19) (2010) 3898-3914. doi: 10.1016/j.jsv.2010.04.009

[35]B.C. Xue, Simulation study on scissor-like element vibrations. The Hong Kong Polytechnic University, MSc Degree Thesis (under supervision of XJ Jing), Dec. 2012.

[36]X.T. Sun, X.J. Jing, J. Xu, L. Cheng, Vibration isolation via a scissor-like structured platform. Journal of Sound and Vibration 333 (9) (2014) 2404-2420. doi: 10.1016/j.jsv.2013.12.025

[37]X.J. Jing, Z.Q. Lang, S.A. Billings, G.R. Tomlinson, Frequency domain analysis for suppression of output vibration from periodic disturbance using nonlinearities, Journal of Sound and Vibration 314 (3) (2008) 536-557. doi: 10.1016/j.jsv.2008.01.031

[38]X.J. Jing, Z.Q. Lang, S.A. Billings, Nonlinear influence in the frequency domain: Alternating series, Systems \& Control Letters 60 (5) (2011) 295-309. doi: 10.1016/j.sysconle.2011.01.003

[39]C.C. Liu, X.J. Jing, S. Daley, F.M. Li, Recent advances in micro-vibration isolation. Mechanical Systems and Signal Processing 56-57 (2015) 55-80. doi:10.1016/j.ymssp.2014.10.007.

[40] XJ Jing, ZQ Lang, Frequency domain analysis of a dimensionless cubic nonlinear damping system subject to harmonic input, Nonlinear Dynamics 58 (3) (2009) 469-485. doi: 10.1007/s11071-009-9493-0.

[41] XJ Jing, ZQ Lang, Frequency domain analysis and design of nonlinear systems based on Volterra series expansion: A parametric characteristic approach, Springer International Publishing, Switzerland, 2015

[42] A. H. Nayfeh, D. T. Mook, Nonlinear Oscillations, New York: Wiley-Interscience, 1979.

[43] CC Liu, XJ Jing, FM Li, Vibration isolation using a hybrid lever-type isolation system with an X-shape supporting structure, International Journal of Mechanical Sciences 98 (2015) 169-177 
[44] Nikolai A. Bobylev, Yurii M. Burman, Sergey K. Korovin, Approximation Procedures in Nonlinear Oscillation Theory, De Gruyter, Sep 1994

Fig. 1 The n-layer SLS-based platform for 2-D base excitations. Solid lines show the original state and dashed lines show the vibration state.

Fig. 2 The motion of a connecting rod in the first layer and geometrical relations. Solid lines for original state and dashed lines for the vibration state.

Fig. 3 The value of first natural frequency $\Omega_{1}$ (solid lines) and second natural frequency $\Omega_{2}$ (dashed lines) for (a) different $k_{e}$; (b) different $n$; (c) different $\theta$.

Fig. 4 Displacement Transmissibilities $T_{x}$ in $(\mathrm{a}, \mathrm{c}, \mathrm{e})$ and $T_{y}$ in $(\mathrm{b}, \mathrm{d}, \mathrm{f})$ of the horizontal and vertical directions with different structural parameters.

Fig. 5 Displacement transmissibilities $T_{x}$ in (a) and $T_{y}$ in (b) for different $c_{1}$ and $c_{2}$.

Fig. 6 (a) A 2-D mass-spring-damper system. (b) Motions of the two springs in horizontal and vertical directions. Solid lines for original state and dashed lines for different vibration states.

Fig. 7 Performance comparisons between the MSD-VI and SLS-based platform

Fig. 8 Performance comparisons between the MSD-VI and SLS platform as $n=4, \theta=\pi / 6$ and $l=1$.

Fig. 9 (a) The QZS-VI system; (b) Deformations of the springs in the QZS-VI system. Solid lines for original state and dashed lines for different vibration positions.

Fig. 10 Comparison between the QZS-VI and SLS platform. The overall performance of the proposed system is better or more preferable in practice both in the horizontal and vertical directions.

Fig. 11 Vehicle seat suspension model. 
Fig. 12 Power spectral densities and time series of the seat suspension as [31] with linear active control device and the n-layer SLS platform for random base excitation; (a) Power spectral density in $x$ direction ( $x$-D) and (c) power spectral density in $y$ direction ( $y$-D); (b) Time series in $x$ direction and (d) time series in $y$ direction.

Fig. 13 The experimental prototype of the SLS-based platform

Fig. 14 Response and transmissibility of the platform for random excitation. (a) The time series accelerate signals of base excitation (Black) and platform (Red); (b) The acceleration transmissibility $T_{a y}$.

Fig. 15 The static deformation for different loading mass $M$ for (a) $\theta=0.8 \mathrm{rad}$, (b) $\theta=0.6 \mathrm{rad}$, and (c) $\theta=0.45 \mathrm{rad}$. The cross in (c) is the static deformation of the prototype

Fig. 16 Responses of the prototype with an initial displacement excitation for two times experiment.

Fig. 17 Comparison between the MSD isolator and the SLS platform.

Table 1 The structural parameters of the 2-D n-layer SLS-based platform and the default values in theoretical analysis.

Table 2 The motion and variables for the SLS-based platform, MSD-VI and QZS-VI 